\title{
Optimisation of cancer drug treatments using cell population dynamics
}

\author{
Frédérique Billy ${ }^{1}$, Jean Clairambault ${ }^{1}$, and Olivier Fercoq ${ }^{2}$ \\ 1 INRIA BANG team, BP 105, F78153 Rocquencourt, and LJLL, UPMC, 4 Place \\ Jussieu, F75005 Paris, France. frederique.billy@inria.fr \\ jean.clairambault@inria.fr \\ 2 INRIA MAXPLUS team, CMAP, Ecole Polytechnique, F91128 Palaiseau, \\ France. olivier.fercoq@inria.fr
}

\section{Introduction}

Cancer is primarily a disease of the physiological control on cell population proliferation. Tissue proliferation relies on the cell division cycle: one cell becomes two after a sequence of molecular events that are physiologically controlled at each step of the cycle at so-called checkpoints, in particular at transitions between phases of the cycle [105]. Tissue proliferation is the main physiological process occurring in development and later in maintaining the permanence of the organism in adults, at that late stage mainly in fast renewing tissues such as bone marrow, gut and skin.

Proliferation is normally controlled in such a way that tissue homeostasis is preserved. By tissue homeostasis we mean permanence in the mean of tissue in volume, mass and function to ensure satisfaction of the needs of the whole organism. In cancer tissues, this physiological control, which also relies on the so-called checkpoints in the division cycle of individual replicating cells, is disrupted, leading to an overproduction of cells that eventually results in the development of tumours.

Anticancer drugs all attack the cell division cycle, either by slowing it down (possibly until quiescence, i.e., non proliferation, cells remaining alive), or by blocking it at checkpoints, which in the absence of cell material repair eventually leads to cell death.

Various mathematical models have been proposed to describe the action of anticancer drugs in order to optimise it, that is to minimise the number of cancer cells or a related quantity, as the growth rate of the cancer cell population. The constraints at stake, met everyday in the clinic of cancers, are related mainly to resistance to treatment in cancer cell populations and to unwanted toxicity in healthy tissues.

We briefly review some of these models, namely ODE models, PDE models with spatial structure, phase structured cellular automata and physiologically 
structured PDE models. We do not claim to be exhaustive in a field where so much has been published in the last fifty years. However, we present the main models used in cancer treatment in the last decades, together with the biological phenomena that can be described by each of them.

We then present some techniques used for the identification of the parameters of population dynamic models used in chemotherapy. We also briefly review theoretical therapeutic optimisation methods that can be used in the context of different models of cell population growth, according to the clinical problem at stake, to the available data and to the chosen model, with their advantages and pitfalls.

We then focus on a novel method of optimisation under unwanted toxicity constraints, presented here in the context of cancer chronotherapeutics. This method is based on optimisation of eigenvalues in an age-structured model of cell population dynamics, the parameters of which can be identified in cell cultures by using recent intracellular imagery techniques relying on fluorescence quantification. Thanks to these fine level quantitative cell observations, the structured cell population model, which takes the cell division cycle into account, gives interesting results for the optimisation of the pharmacological treatments of cancer.

Finally, bases for cell population dynamic models, with external control targets, that ought to be used to physiologically represent the effects of different anticancer drugs in use in the clinic, are sketched, as are possible schemes for multitarget multidrug delivery optimisation, designed to meet present clinical challenges in everyday oncology.

\section{Drugs used in cancer treatments and their targets}

Although these are important co-occurring phenomena in cancer and make all its malignancy, tissue invasion and the development of distant metastases will not be mentioned here, for the simple reason that no efficient treatments exist against them specifically so far. For instance, matrix metalloprotease inhibitors (anti-invasive agents) were tested in the past, even until phase II clinical trials, but their development has been arrested in particular due to high toxicity. We will thus stick to drugs that impact on local cancer growth, a process which itself is initiated by growing impairment of the normal physiological control on the cell division cycle in cell populations.

From this point of view, drugs used in cancer treatments may be roughly divided into those that drive cells to their death, which we define here as cytotoxic, and those that just slow down the cell division cycle, letting cells alive, but containing teumour development, which we will define here as cytostatic. Note that 'cytotoxic' and 'cytostatic' are terms on which consensus is not so widespread, hence this necessary precision for our purposes. 


\subsection{Fate of drugs in the organism: molecular pharmacokinetics- pharmacodynamics}

Anticancer drugs are delivered into the general circulation, either directly by intravenous infusion, or indirectly by oral route, intestinal absorption and enterohepatic circulation (i.e., entry in the general blood circulation from the intestine via the portal vein towards the liver, and possibly back from the liver to the intestine via bile ducts). Their fate, from introduction in the circulation until presence of an active metabolite in the neighbourhood of their intracellular targets, can be represented by pharmacokinetic (PK) compartmental ordinary differential equations (ODEs) for their concentrations. It is also theoretically possible to represent this fate by spatial partial differential equations (PDEs) with boundary conditions instead of exchange rules between compartments when data on spatial diffusion of the drugs and some geometry of their distribution domain is known - but this is seldom the case.

Then, in the cell medium, either an individual cell, or a mean intracellular medium in a population of cells, pharmacodynamic ( $P D)$ differential equations must be used to relate local drug concentrations with molecular effects on their targets. At this level of description, it is a priori more relevant to describe by physiologically structured than by spatially structured models the population of cells under pharmacological attack, since anticancer drugs act mainly by blocking the cell division cycle, which does not give rise to a spatially structured cell population (apart from the very early stages of avascular spheroid tumour growth, little geometry is relevant to describe a tumour seen under the microscope).

\subsection{Cytotoxics and cytostatics}

Driving cells to their death may be obtained either by damaging the genome, or more indirectly by impairing essential mechanisms of the cell division cycle, such as enzymes thymidylate synthase (an enzyme that plays an essential role in DNA synthesis and is one of the main targets of cytotoxic drug 5-FU) or topoisomerase I (another essential enzyme of DNA synthesis, target of cytotoxic drug irinotecan). The resulting damaged cell, unable to proceed until division into two viable cells, is normally blocked at one or the other checkpoint, mainly $G_{1} / S$ or $G_{2} / M$ (recall that the cell division cycle is classically divided into 4 successive phases, $G_{1}, S$ for DNA synthesis, $G_{2}$, and $M$ for mitosis).

Then, unless it may be repaired by specific enzymes - that are often overexpressed in cancer cells -, these impaired cells, blocked at a checkpoint, are subsequently sent to 'clean death' by the physiological mechanism of apoptosis (also possibly impaired in cancer, resulting in abnormal cells bypassing these checkpoints). As mentioned above, we define here this class of drugs, that have for their ultimate mission to kill cancer cells - even if their primary 
action is not to directly damage the genome, but rather to damage cell cycle enzymes - as cytotoxics.

We reserve the term cytostatic to those non cell-killing drugs that merely slow down proliferation, usually by maintaining cells in $G_{1}$ with possible exit to $G_{0}$, that is by definition the quiescent phase, i.e., the subpopulation of cells that are not committed in the cell division cycle. Indeed, before the restriction point inside $G_{1}$, cells may stop their progression in the cell cycle and go back to quiescence in $G_{0}$. This last category comprises all drugs that act as antagonists of growth factors, which may be monoclonal antibodies or tyrosine kinase inhibitors.

\subsection{Drugs that act on the peritumoral environment}

We might also attach to this 'cytostatic' category drugs that act on the tumour environment, such as for instance anti-angiogenic drugs (that impoverish it in oxygen and nutrients) or molecules that could be able to modify local $\mathrm{pH}$ in a sense unfavorable to cancer cells, assuming that their action is not to entrain cell death, but to indiectly slow down progression in the cell cycle, which is possible mainly, if not only, in the $G_{1}$ phase.

Acting on cancer cell populations without killing any of these cells but only by limiting their thriving, even inducing their decay, by unfavorable environmental conditions is certainly an ideal therapy, which avoid cell killing also in healthy cell populations, and it has been achieved in some rare cases in monotherapy, e.g., by Imatinib (initially known as STI571) for Chronic Myelogenous Leukaemia [53, 100]. Yet most treatments of cancer use combinations of cytotoxics and cytostatics (e.g., irinotecan and cetuximab [44]), for non cell-killing therapeutics alone are seldom sufficient in advanced stages of cancers.

Another instance of such combination of cell-killing and non cell-killing therapeutics is the treatment of Premyelocytic Acute Myeloblastic Leukaemia (APL or AML3) by combining an anthracyclin and a redifferentiating agent (for AML is characterised by a blockade of differentiation in non proliferating hematopoietic cells, and redifferentiating agents such as All-TransRetinoic Acid (ATRA) lift such blockade in the case of APL) [66]. Here, maturation, and not proliferation (i.e., not the cell cycle, which is not affected by them), is the target of redifferentiating agents, which may thus by no means be considered as cytostatic.

\subsection{Representation of drug targets}

It is appropriate to consider anticancer drugs, cytotoxic or cytostatic, and their targets, through their effects on the cell cycle in cell populations. This of course assumes that a model of the cell cycle in a proliferating cell population is given. Nevertheless we also firstly mention other models, which either do not include the cell cycle or do not describe events at the level of a cell population. 
In a review article [75], Kimmel and Świerniak considered two possibilities to represent in a mathematical model the action of cytotoxic drugs on their targets in a proliferating cell population: either by a possible direct effect on cell death, enhancing it, presumably by launching or accelerating the apoptotic cascade in one or more phases of the cell cycle, or by a blockade of one or more transitions between two phases, arresting the cycle at some checkpoint, most often with the involvement of protein p53, and only secondarily launching cell death.

This is indeed a general alternative in the representation of the effects of cytotoxics. If no cell cycle phase structure has been put in the population dynamic model used to represent the evolution of the cell populations at stake, i.e., when no account is taken of cell cycle phases in these populations, then only the first possibility exists: modulation of a death term.

As regards cytostatics (which by definition are not supposed to kill cells, at least not directly), the representation of their action in physiological models with age structure for the cell cycle should be somewhat different. It can be done either by a slowdown of the progression speed in the $G_{1}$ phase (or in the proliferating phase in a one-phase model) or by an action on the exchanges between non proliferating $\left(G_{0}\right)$ and proliferating phases when a $G_{0}$ phase is represented in the model.

It is also possible to combine cytostatic and cytotoxic effects in the same model. In [69], for instance, the authors use an age-structured model with a 1phase proliferative subpopulation exchanging cells with a nonproliferative cell compartment to combine a slowdown effect on proliferation for the cytostatic effect with an increase in the cell death term for the cytotoxic effect - of the same drug, lapatinib, a tyrosine kinase inhibitor, in their case, the variation between these effects depending on the dose. Acting on two different targets in a cell cycle model by two different drugs, a cytotoxic and a cytostatic one, in the same cell population is thus possible, and such models are thus amenable to study and optimise combination therapies, such as cetuximab+irinotecan advocated in [44].

\section{Overview of cell population dynamics designed for cancer treatment}

In this section, we present an overview of several kinds of cell population models that have been used in modelling anticancer treatments. Some works we refer to do not include cancer treatment optimisation, but it is important to mention them as they could be used as a first step towards cancer therapy optimisation. As so much has been published in the last fifty years, we do not claim to be exhaustive, only recollecting the main models used to describe the fate of cell populations submitted to cancer treatments. 


\subsection{ODE models for growing cell populations with drug control}

The first models of tumour growth were developed to reproduce and explain experimentally observed tumour growth curves. The most common ones are the exponential model $\left(\frac{d N}{d t}=\lambda N\right)$, the logistic $\left(\frac{d N}{d t}=\lambda N\left(1-\frac{N}{K}\right)\right.$, where $K$ is the maximum tumour size, or "carrying capacity" of the environment), and the Gompertz $\left(\frac{d N}{d t}=\lambda N \ln \left(\frac{K}{N}\right)\right.$, where again $K$ is the carrying capacity). Contrary to the exponential model, the logistic and the Gompertz models take into account the possible limitation of growth due, for instance, to a lack of space or resources, assuming that the instantaneous growth rate $\frac{d N}{N d t}$ depends on the carrying capacity of the environment. The Gompertz model was initially developed in the context of insurance [62] and was first used in the nineties to fit experimental data of tumour growth [81]. A lot of studies on drug control are based on these models [14, 15, 35, 96, 97, 98, 106, 107, 108]. For instance, Murray $[106,107]$ considered a two-population Gompertz growth model with a loss term to model the effect of the cytotoxic drug. He considered both tumour and normal cells in order to take into account possible side-effects of the treatment on the population of normal cells. Murray's purpose was to minimise the size of the population of tumour cells at the end of the treatment while keeping the population of normal cells above a given threshold. In [108], Murray took into account cell resistance to chemotherapy and applied the problem of optimising drug schedules to a two-drug chemotherapy. In [96], Martin developed a model to determine chemotherapy schedules that would minimize the size of the tumour at the end of the treatment, under constraints of maximal drug doses (individual doses and cumulative dose), ensuring that the tumour decrease might be faster than a given threshold. In further works, Martin et al. also introduced tumour cell resistance to chemotherapy [97, 98].

More recently, one of us and his co-workers $[15,35]$ investigated the effects of oxaliplatin on tumour cells and healthy cells. To model tumour growth, they used a Gompertz model modified by a "therapeutic efficacy term" as a death term depending on the drug concentration.

In [35], the work presented in [15] is extended by coupling this model of tumour growth to a model of healthy cell growth and to a three-compartment model describing the time evolution of the concentration of oxaliplatin in plasma, healthy tissue and tumour tissue. This work was done on the basis of experimental data related to oxaliplatin PK-PD and tumour growth curves with or without drug injection to determine the model parameters and compared time-scheduled infusion schemes with constant ones. In the same work, the drug infusion schedule was optimised by determining drug infusion patterns that should maximise tumour cell death under the constraint of minimising healthy cell death. Barbolosi and Iliadis $[14,70]$ coupled a Gompertz model of tumour growth, perturbed by a cytotoxic efficacy term, to a two-compartment model of the chemotherapy PK (plasmatic and active 
drug concentration). They investigated optimal drug delivery schedules under constraints of maximal allowed drug (single doses and cumulative dose) and leukopenia.

In an attempt to design a more realistic model of tumour growth under angiogenic stimulator and inhibitor control, Hahnfeldt et al. [67] proposed a two-variable model derived from the Gompertz model. It is based on observations made on experimental data from lung tumours in mice treated by an antiangiogenic drug. Hahnfeldt et al. introduced as a variable the carrying capacity of the environment, $K$ :

$$
\begin{aligned}
& \frac{d N}{d t}=\lambda N \ln \left(\frac{K}{N}\right) \\
& \frac{d K}{d t}=b N-\left(\mu+d N^{2 / 3}\right) K-\eta g(t) K
\end{aligned}
$$

where $b$ is the rate of the tumour-induced vasculature formation, $\mu+d N^{2 / 3}$ represents the rate of spontaneous and tumour-induced vasculature loss, $g(t) \geq 0$ represents the antiangiogenic drug concentration.

This model enables to take into account the vasculature, that provides nutrients and oxygen to tumour cells, and thus to study the effects of several anti-angiogenic factors on tumour growth. D'Onofrio et al., based themselves on this model, proposed different expressions for $K$, modelling for instance endothelial cell proliferation or delayed death, to investigate the action on tumour growth of anti-angiogenic therapies [46, 47, 48] and of combined therapies [49]. In the same way, Ledzewicz et al. [83], basing themselves on [55], considered the following model

$$
\begin{aligned}
& \frac{d N}{d t}=-\lambda N \ln \left(\frac{N}{K}\right)-\varphi v N \\
& \frac{d K}{d t}=b K^{2 / 3}-d K^{4 / 3}-(\mu+\gamma u-\eta v) K
\end{aligned}
$$

where $u$ and $v$ represent the doses of an anti-angiogenic drug and of a cytotoxic drug, respectively, and $\varphi, \gamma, \eta$ their effects on tumour cells and on vasculature. The authors introduced an optimisation problem to minimise the tumour cell mass under constraints on the quantity of drug to be delivered.

Most cancer chemotherapies are cell cycle phase-specific, which means that they act only on cells that are in a specific phase of the cell division cycle, for instance in $S$ or in $M$. To take into account such specificities, models describing the cell cycle have been designed. Without always entering into the details of cell cycle phases, several models distinguish between proliferating and nonproliferating cells $[114,115,139,140]$. They take into account two kinds of cell populations, proliferative and quiescent, by representing time variations of their densities, and allow exchanges of cells from one population to the other. They are based on the fact that only proliferative cells are sensitive 
to chemotherapies and they allow to study the effect on tumour growth of several treatment schedules and to determine the optimal ones. For instance, in $[114,115]$, Panetta et al. proposed the following model

$$
\frac{d}{d t}\left(\begin{array}{l}
x_{1} \\
x_{2}
\end{array}\right)=\left(\begin{array}{cc}
\alpha-\mu-\eta & \beta \\
\mu & -\beta-\gamma
\end{array}\right)\left(\begin{array}{l}
x_{1} \\
x_{2}
\end{array}\right)
$$

where $x_{1}$ and $x_{2}$ represent the cycling and non-cycling tumour cell mass respectively, $\alpha$ the cycling growth rate, $\mu$ the rate at which cycling cells become non-cycling, $\eta$ the natural decay of cycling cells, $\beta$ the rate at which noncycling cells become cycling, and $\gamma$ the natural decay of non-cycling cells. All these parameters are supposed to be constant and positive. By adding a drug-induced death term in the equation on cycling cells, the authors investigated the effects on tumour growth of two kinds of periodic chemotherapies: a pulsed one and a piecewise continuous one. They also considered the effect on a population of non tumour cells (or normal cells) in order to determine optimal drug schedules. Some authors later based themselves on this model to determine optimal chemotherapy schedules [56, 85]. Using experimental data, Ribba et al. [124] introduced a third kind of cells, the necrotic cells, and the carrying capacity in order to investigate the effect of an antiangiogenic treatment on tumour growth dynamics and on hypoxic and necrotic tissues within the tumour.

Kozusko et al. [80] deepened the work of Panetta et al. [114] by developing a model of tumour growth integrating two compartments within the cell cycle: one for cells in phases $G_{1}$ and $S$ and another for cells in $G_{2}$ and $M$. They based their model on experimental data to represent the effect on tumour growth of an antimitotic agent (curacin A), that prevents cells from dividing. They modeled the blockade of cells in the $G_{2} / M$ phase of the cell cycle according to the treatment dose, and distinguished resistant cells from sensitive ones. This model was able to predict a minimum dose of treatment able to stop growth of both kinds of cells. To analyse the effect on tumour growth of another anticancer treatment (mercaptopurine) according to varying degrees of cell resistance, Panetta et al. [116] modified the model introduced by Kozusko et al. [80] by distinguishing phases $G_{0} / G_{1}, S$ and $G_{2} / M$.

\subsection{PDE models with spatial dynamics for tumour growth and drug effects}

ODE models presented above do not integrate any spatial dimension. They were historically developed to explain in vitro tumour growth curves. Obviously in vivo tumour growth depends on its environment. For instance, it depends on the mechanical properties of the supporting tissue, on the local quantity of nutrients and oxygen, on the local concentrations of pro and antigrowth chemical factors, etc. PDE models integrating spatial dynamics are thus better suited than ODE models for the design of realistic models of tumour growth. Greenspan [63] was the first author to take into account the 
spatial dynamics of tumour cells and oxygen through the simplifying hypothesis of a spherical symmetry of the diseased tissue (tumour spheroid). Several models are based on his [33, 34, 43, 45, 101, 127].

Reaction-diffusion PDEs are best suited to describe the space and time evolution of the concentrations of chemical substances and of cell densities. Such equations allow to take into account the interactions of a diffusing chemical molecule or of a population of cells with its environment.

Thus Swanson et al. [131, 132, 133] used the classical KPP-Fisher model that is frequently used to represent the spatial progression of so-called "travelling waves" (see [103] for details), to develop a model of brain tumour growth that takes into account tumour cell proliferation and diffusion

$$
\frac{\partial p}{\partial t}=\nabla \cdot(D(x) \nabla p)+\rho p(1-p)
$$

where $p$ is the tumour cell density (that depends on space and time), $D(x)$ the diffusion rate (that depends on space), $\rho$ the net proliferation rate. In fact, its linearised form around the origin

$$
\frac{\partial p}{\partial t}=\nabla \cdot(D(x) \nabla p)+\rho p
$$

is sufficient to describe tumour progression (and it has an analytic solution if $D(x)$ is constant). The difficulty here resides in the identification of the diffusion coefficient $D(x)$, which is in fact far from constant, since it depends on the nature of cerebral matter, grey or white, and the brain is not known to posses a simple spatial structure.

In [132], Swanson et al. modelled the action of a chemotherapy by introducing in Equation (6) a linear death term that also depends on space and time. They investigated drug delivery according to the tissue heterogeneities of the brain (white or grey matter).

Competition between cells for the gain of space and nutrients influences tumour growth. Thus, on the basis of a Lotka-Volterra type model [109, 110], Gatenby et al. [59, 60] modelled competition between healthy and tumour cells to phenomenologically represent the mutual negative influences of the populations on each other. They highlighted the limitations of the clinical cytotoxic strategies that solely focus on killing tumour cells and not on preserving healthy cell populations from toxic side effects of the anticancer drugs.

Cell proliferation and cell death induce changes in the tumour volume. This phenomenon has to be taken into account to model tumour cell growth in a more mechanistic way. It is usually done by adding a transport term in the left-hand side of the reaction-diffusion equation

$$
\frac{\partial p}{\partial t}+\nabla \cdot(\mathbf{v} p)=\nabla \cdot(D \nabla p)+f(p)
$$


where $f$ represents the reaction term, and $\mathbf{v}$ the velocity of the transport movement. The velocity $\mathbf{v}$ can be determined by using Darcy's law and, for instance, assumptions on the total amount of cells.

Authors generally consider that the population of tumour cells is submitted to a growth signal representing all growth signals (inhibitors or promoters). The equations governing the evolution of the density (or mass) of tumour cells and of the concentration of chemicals are derived by applying the principle of mass conservation to each species. The common form of such equations is

$$
\begin{aligned}
& \frac{\partial p}{\partial t}+\nabla \cdot(\mathbf{v} p)=\nabla \cdot\left(D_{p} \nabla p\right)+\alpha_{p}(c, p)-\delta_{p}(c, p) \\
& \frac{\partial c}{\partial t}=\nabla \cdot\left(D_{c} \nabla c\right)+\alpha_{c}(c)-\delta_{c}(c, p)
\end{aligned}
$$

where $p$ is the density of tumour cells, $c$ the concentration of the chemical (nutrients, oxygen, ...), $D_{p}$ the diffusion rate of tumour cells, $\alpha_{p}$ represents their proliferation rate, $\delta_{p}$ their spontaneous death rate, $D_{c}$ is the diffusion rate of the chemical, $\alpha_{c}$ represents its production rate, $\delta_{c}$ its degradation rate. Because tumour cell proliferation and death depend on the concentration of the chemical, functions $\alpha_{p}$ and $\delta_{p}$ depend, for instance linearly, on the concentration of the chemical and on the tumour cell density. The function $\alpha_{c}$ depends, for instance linearly, on the concentration of the chemical and the function $\delta_{c}$ depends both on the chemical concentration and on the density of tumour cells to model, for instance, the consumption of the chemical substance by tumour cells. The same kind of equation as Equation (10) can be used to describe the evolution of the concentration of drug $u$.

As already mentioned in Section 2, cancer therapies can have different effects on tumour growth. To model the effect of a drug inducing tumour cell death (cytotoxic), one can add in the right-hand term of Equation (9) a death term; thus the equation governing the density of tumour cells submitted to the effect of a cytotoxic drug may be given by

$$
\frac{\partial p}{\partial t}+\nabla \cdot(\mathbf{v} p)=\nabla \cdot\left(D_{p} \nabla p\right)+\alpha_{p}(c, p)-\delta_{p}(c, p)-K_{\text {cyto }}(u, p)
$$

where $K_{\text {cyto }}$ is a positive function that depends on the drug concentration and on the tumour cell density, Equation (10) remaining unchanged.

Instead, to model the effect on tumour growth of an anti-angiogenic therapy that will reduce oxygen supply, one can add such a decay term in the right-hand term of Equation (10), which then becomes

$$
\frac{\partial c}{\partial t}=\nabla \cdot\left(D_{c} \nabla c\right)+\alpha_{c}(c)-\delta_{c}(c, p)-K_{\text {angio }}(u, c)
$$

where $K_{\text {angio }}$ is a positive function that depends on the drug concentration and on the chemical concentration; in this case, Equation (9) remains unchanged.

Thus, in [73], Jackson et al. considered a model with two kinds of cells differing by their sensitivity to a cytotoxic treatment: one cell type was less 
sensitive than the other one. They assumed that the tumour was a spheroid, thus reducing the dimension from three to one, using radial symmetry. The drug fate was modelled through the variations of its tissue concentration, via a term of blood-to-tissue transfer (the drug concentration in blood being prescribed by the therapy scheduling). The authors compared the tumour response to an equal amount of drug administered either by bolus injection or by continuous infusion. Jackson based herself on this work to develop a model of the action of an anti-cancer agent (doxorubicin) on tumour growth [72]. This model is composed of a submodel of tumour growth coupled to a three-compartment submodel of intratumour drug concentration (extracellular space, intracellular fluid space, nucleus space) and to a submodel of the plasma concentration of the drug. The intracellular action of the drug on tumour cells is modelled through a Hill-type function. This model allows to study the tumour response to repeated rounds of chemotherapy.

In [112], Norris et al. investigated the effects of different drug kinetics (linear vs. Michaelis-Menten kinetics) and different drug schedules (single infusion vs. repeated infusions) on tumour growth.

Frieboes et al. [57] developed a mathematical model of tumour drug response that takes into account the local concentration of drug and nutrients. The authors considered two cell phenotypes, viable and dead tumour cells, and supposed that their mitosis and apoptosis rates depended on the nutrients and drug concentration. This model was calibrated on in vitro cultures of breast cancer cells.

More mechanistic (i.e., more molecular than purely phenomenological) models have been used to take into account details of the angiogenic process. Endothelial cells, that constitute the blood vessel wall migrate towards a gradient of a chemoattractant substance secreted by quiescent (or hypoxic) tumour cells (this movement is termed chemotaxis). Continuous models of angiogenesis usually take into account the density of endothelial cells and the tissue concentration of the chemoattractant substance. They are based on the following equations

$$
\begin{aligned}
\frac{\partial m}{\partial t} & =\nabla \cdot\left(D_{m} \nabla m\right)+\alpha_{m} m-\chi_{m} \nabla \cdot(m \nabla w)-\delta_{m} m \\
\frac{\partial w}{\partial t} & =\nabla \cdot\left(D_{w} \nabla w\right)+\alpha_{w}(q) w-\delta_{w} w
\end{aligned}
$$

where $m$ denotes the density of endothelial cells, $D_{m}$ their diffusion rate, $\alpha_{m}$ their proliferation rate, $\chi_{m}$ their chemotaxis rate, $\delta_{m}$ their death rate, $w$ the concentration of the chemoattractant substance, $D_{w}$ its diffusion rate, $\delta_{w}$ its production rate that depends on the density of quiescent tumour cells $q, \delta_{w}$ its degradation rate. Such models can also include other kinds of cells or chemical substances, see for instance [32] for a review of angiogenesis models.

The coupling of angiogenesis models to tumour growth models is usually done via the concentration in oxygen (or nutrients), the time and space evo- 
lution of which is given by a reaction-diffusion PDE based on the fact that oxygen is delivered by the vasculature and mostly consumed by tumour cells. This coupling enables to describe in a more realistic way the effects of antiangiogenic therapies on tumour vasculature and thus on tumour growth.

Thus, Sinek et al. [128] based on the model of vascular tumour growth developed by Zheng et al. [141] and on experimental data to develop a model of tumour growth and vascular network coupled to a multi-compartment pharmacokinetic-pharmacodynamic (PK-PD) model. Their purpose was to analyse the effect on tumour growth of two anti-cancer drugs, doxorubicin and cisplatin (compartments of the PK-PD model were drug-specific). They concluded that drug and oxygen heterogeneities, possibly due to irregularities of the vasculature, can impact drug efficacy on tumour cells. Kohandel et al. [79] proposed a model that also takes into account tumour cells, the tumour vascular network and oxygen to investigate the effect on tumour growth of different schedules of single and combined radiotherapy and anti-angiogenic therapy.

\subsection{Phase-structured cellular automata for the cell division cycle and drug effects}

Drug effects on tumour growth can also be investigated by means of phasestructured cellular automata to represent the cell division cycle. Cellular automata enable to describe individual cancer cell evolution within a population of cells. Thus Altinok et al. developed a cellular automaton for the cell cycle $[3,4,5,6]$. This automaton does not take into account molecular events but phenomenologically describes cell cycle progression. The states of this automaton correspond to the phases of the cell cycle. Transition between two states of the automaton correspond to cell progression through the cell cycle, or exit from the cell cycle, and are supposed to respect some prescribed rules. For instance each phase of the cell cycle is supposed to be characterised by a mean duration and a variability in order to take into account inter-cell variability that can appear within a population. This model enables to study, on a whole population of cells, the impact of the variability in the duration of the cell cycle phases on cell desynchronisation through the cell cycle.

Such modelling is motivated by the fact that one way to optimise pharmacological treatments in cancer, taking into account of the cell division cycle on which tissue proliferation relies, is to take advantage of the control that circadian clocks are known to exert on it. Such treatments are termed chronotherapies of cancer $[87,88,89,90,91,92]$. In order to investigate the effects of chronotherapy on the growth of a tumour cell population, Altinok et al. coupled this cellular automaton with a model of the circadian clock through kinases known to induce or inhibit the transition from $G_{2}$ to $M$. For instance, in $[4,5]$, the authors were interested in the action of 5-fluorouracil (5-FU), an anticancer drug known to block cells in the $S$ phase. They modelled the effects of this drug by increasing the probability that cells submitted to 5 -FU 
while in $S$ phase exit from the cell cycle at the next $G_{2} / M$ transition. They compared the cytotoxic efficacy of continuous administration of 5-FU and of several chronomodulated therapies that differed from their administration peak time. Later, in [6], Altinok et al. analysed the cytotoxic effects of 5-FU chronotherapies according to their administration peak time and to the cell cycle mean duration. As they did for 5-FU, Altinok et al. also investigated the effects of oxaliplatin chronomodulated therapies on tumour cells. Contrary to 5 -FU, oxaliplatin is an anticancer agent that is not phase-specific. Therefore the authors modelled the effects of oxaliplatin in a non phase-specific way, by increasing the probability for exposed cells of exiting the cell cycle at the next checkpoint $\left(G_{1} / S\right.$ or $G_{2} / M$ transitions).

\subsection{Physiologically structured PDE models for the cell cycle and drug effects}

Time and space are not the only two variables on which tumour growth depends. In fact, tumour growth also depends on the physiological properties of cancer cells, that can be for instance age of the cells (i.e., time since the last cell division), mass or volume of the cells, or their DNA content. To take this phenomenon into account, the McKendrick PDE framework is the best suited

$$
\begin{cases}\frac{\partial n}{\partial t}(a, t)+\frac{\partial}{\partial a}[g(a) n(a, t)]+d(a) n(a, t)=0, & \left(t>0, a>a_{\text {min }}\right), \\ n\left(a_{\text {min }}, t\right)=\int_{a_{\text {min }}}^{\infty} \beta(s) n(s, t) d s, & (t>0), \\ n(a, 0)=n_{0}(a) . & \left(a>a_{\text {min }}\right),\end{cases}
$$

where $n(a, t)$ is the density of tumour cells with the characteristic $a$ (age, mass, volume, DNA content, etc) at time $t, g$ is the tumour growth rate, $d$ is the death rate, $\beta$ is the tumour cell birth rate, $a_{\text {min }} \geq 0$ is the minimum value of $a$. Note that $g, d, \beta$ depend on $a$.

Physiologically structured cell population dynamics models have been extensively studied in the last 25 years, see e.g. [7, 8, 9, 13, 16, 20, 26, 39, 40, 65, $69,71,74,103,123,138]$. For instance, Iwata et al. [71] developed a model of the dynamics of the colony size distribution of metastatic tumours, assuming that both primary and metastatic tumour growth depended on the size of the tumour. The authors proposed a Gompertz equation to model the primary tumour growth and a McKendrick type equation to model the evolution of the colony size distribution of metastases. Kheifetz et al. [74] proposed a model for tumour cell age distribution to investigate tumour cell dynamics under periodic age-specific chemotherapy. Hinow et al. [69] developed an agestructured PDE model to investigate the cytotoxic and cytostatic effects on tumour growth of a cancer drug, lapatinib, on the basis of biological experiments. The authors distinguished between proliferative and non-proliferative cells and assumed that only proliferative cells were ageing. 
One of us with his co-workers $[26,39,40]$ considered a multiphase agestructured PDE model in which they introduced time dependency of the parameters (death rate, transition rate from one phase of the cell cycle to the next one). They investigated the effects of a circadian control on the tumour growth rate with and without a periodic cell cycle phase specific chronotherapy. More details about [26] can be found in Section 7 .

Basse et al. $[17,18,19]$ developed a phase- and size-structured model of a cell population submitted to paclitaxel, a cancer agent that induces mitotic arrest of the cell cycle and cell death. The size of the cell was considered as determined by its DNA content and some of the model parameters were determined by fitting experimental flow cytometry data. On the basis of the work of Spinelli et al. [130], Basse et al. [20] developed a phase- and age-structured model of a cell population submitted to a chemotherapy. They considered several cancer agents and assumed that these chemotherapies affected tumour cell population dynamics by modifying cell cycle phase transition functions or by killing cells in the mitotic phase.

Webb [138] proposed a both age- and size-structured model for normal and tumour cell dynamics under chemotherapy, on the basis of the McKendrick model with an additional transport term. He supposed that the two population differed by their mean cell cycle duration, that was longer for tumour cells, and modelled the effects of the chemotherapy by a time periodic death term. His aim was to take the resonance phenomenon into account to determine optimal period of the cancer treatment in order to induce the lowest tumour growth rate and the highest normal cell population growth rate.

Finally, we present some examples of models that are not actually physiologically structured PDE models but that derive from them.

Ubezio and co-workers [94, 95, 104, 135] based themselves on an age and phase structured PDE model to develop a discrete age-structured model of cell cycle describing the time evolution of the number of cells of age $a$ at time $t$ in the phases $G_{1}, S$ and $G_{2} / M$ of the cell cycle. This model also takes into account the inter-cell variability in phase duration. The potential effects of drug (blocking cells in $G_{1}$ or in $G_{2}$, etc) were modelled through separate parameters. Thanks to this model and experimental data, Montalenti et al. [104] investigated the effects of several doses of cisplatin on ovarian carcinoma cells. Although cisplatin is known to block cells mostly (but not only, since as an alkylating agent inducing double strand breaks throughout the cell cycle, it is not phase-specific) in $G_{2}$, they also analysed the effects of cisplatin on cells in $G_{1}$. In [94, 95], based on experimental data, Lupi et al. investigated the effects of topotecan and melphalan respectively, on ovarian cells. Later, Ubezio et al. [135] deepened the previous works of their team by examining the effects of five drugs (doxorubicin, cisplatin, topotecan, paclitaxel and melphalan) on ovarian cancer cells using several doses.

Delay differential models can also be viewed as deriving from age-structured PDE models since they can be obtained by integrating PDEs along char- 
acteristics. Thus Bernard et al. [23] proposed a model composed of delayed differential equations to model tumour and normal cell population dynamics in the phases of the cell cycle under circadian control and chemotherapy. They compared the efficacy and toxicity of constant and chronomodulated schedules of 5-FU, a phase-specific drug used in the treatment of colorectal cancer.

\subsection{Mixed models, both spatially and physiologically structured}

We call "mixed models", models that include both spatial and physiological dynamics. Such models are useful to investigate spatial changes induced by a phase-specific chemotherapy combined or not with an antiangiogenic agent. This kind of models has not been highly developed. Bresch and co-workers $[27,30,31]$ developed a multiscale model of tumour growth that includes cell age in the proliferative phases of the cell cycle and tissue motion of tumour cells. On the basis of the model developed by Bresch et al., coupled with an angiogenesis model, one of us et her co-wokers [27] investigated the effects of an innovative antiangiogenic drug on tumour vasculature and hence on tumour growth. This multiscale model takes into account some molecular events such as cell cycle dynamics and cell receptor binding. This model could be coupled to a model of phase-specific drug, such as $5-\mathrm{FU}$, to analyse tumour and endothelial cell dynamics under drug infusion. It could also be interesting to determine optimal drug schedules that would maximise tumour cell death under constraints of minimising endothelial cell death to ensure drug delivery to tumour cells (remember that endothelial cells are cells that constitute the vessel wall, see Section 3.4 for details).

Alarcón et al. [2] proposed a more complex multiscale model of vascular tumour growth that integrates tissue, cell and intracellular scales. For instance, this model accounts for vascular network, blood flow, cell-cell interaction, cellcycle, VEGF production and integrates several kinds of models (ODE models, cellular automata, etc). The authors investigated the effects of low and high concentrations of protein p27 on the dynamics of tumour and normal cell populations.

\section{The control and its missions: representing the action of drugs}

In the previous section, we have described some dynamic models for cell populations frequently used in the study of cancer growth and treatments. These models can thus be seen as controlled dynamic systems with drug effects as their control functions. Various examples of such drug effects have been given in Section 2. Introducing pharmacokinetics (i.e., evolution of concentrations) for the drugs chosen produces additional equations to the cell population dynamic model, and their pharmacodynamics (i.e., actual drug actions) modify 
this cell dynamics according to the target and to the effect of the drugs. Then, optimisation of cancer treatments can be represented as an optimal control problem on this controlled dynamic system. In this section, we first discuss how the drug infusions are taken into account in the model, then we give examples of objective functions and constraints considered in the literature on the treatment of cancers.

\subsection{Classes of control functions: what is fixed and what may be optimised}

We introduce a vector space $X$, called the state space. At each time $t \in \mathbb{R}_{+}$, the state of the system is $x(t) \in X$. This variable lists all the data necessary to represent the system. It should at least contain the number (or density) of cells for each type considered. The state may have coordinates for healthy and cancer cells and for each phase considered. In a PDE model, the state may also distinguish between ages or between locations of cells. In the PDE case, $X$ has infinite dimension. The state should also contain concentrations of drugs in each compartment of the pharmacokinetic model.

We denote by $u$ the control function, $u: \mathbb{R}_{+} \rightarrow U$. It represents the (multi)drug infusion schedule time by time, one coordinate per drug. The dynamics of the biological system can thus be written as

$$
\dot{x}(t)=f(t, x(t), u(t)),
$$

where $f: \mathbb{R}_{+} \times X \times U \rightarrow X$. Given a control $u(\cdot)$, under standard hypothesis on the dynamics $f$, the state is uniquely defined and we will denote the state variable associated to the control $u(\cdot)$ by $x_{u}(\cdot)$. Examples of such functions are given in Section 3, for instance in Equations (1)-(4), (11)-(12) and (15).

Alternatively, instead of a control function, one may consider simpler predefined infusion schemes with only a small number of control parameters. Such infusion schemes may represent either a simple model for an early study or a consequence of technical constraints such as the fact that oral drugs can only be administered at fixed hours (at meal time for instance). Then $u \in \mathbb{R}^{m}$ is a set of parameters and the dynamics is $\dot{x}(t)=f_{u}(t, x(t))$. Examples of such parameters are the period of a periodic scheme $[115,138]$ or the phase difference between a circadian clock and the time of drug infusion initiation [6] (see also Section 3).

\subsection{Objective functions: measuring the output}

An optimisation problem consists in maximising or minimising a given realvalued objective function, that models the objective we want to reach.

The main purpose of a cancer treatment is to minimise the number of cancer cells. When the model takes into account the number of cancer cells directly $[6,15,49,56,75,84,118,136]$, the objective function is simply the 
value of the coordinate of the state variable corresponding to the number of cancer cells at a time $T, T$ being either fixed or controlled. To take into account the drug effects, Swierniak et al. [75, 134] defined a performance index to minimise the number of tumour cells at the end of the treatment while minimising the cumulated drug dose (viewed as a measure of the cumulated drug effects on healthy cells).

The optimisation problem can also be formalised as the minimisation of the asymptotic growth rate of the cancer cell population $[26,114,138]$. Hence, the number of cancer cells will increase more slowly, or even eventually decrease. We will present this approach in a linear frame (hence controlling eigenvalues) in Section 7.

Alternatively, in [134], Swierniak et al. discussed the problem of maximising both the final number of normal cells and the cumulated drug effects on tumour cells. They concluded that this approach led to optimisation principles similar to those developed to solve the problem of minimising both the final number of tumour cells and the cumulated drug effects on healthy cells.

\subsection{Constraints, technological and biological, static or dynamic}

\section{Toxicity constraints}

A critical issue in cancer treatment is due to the fact that drugs usually exert their effects not only on cancer cells but also on healthy cells. A simple way to minimise the number of cancer cells is to deliver a huge quantity of drug to the patient, who is however then certainly exposed at high lethal risk. In order to avoid such "toxic solutions", one may set constraints in the optimisation problem, which thus becomes an optimisation problem under constraints.

Putting an upper bound on the drug instantaneous flow [56] and/or on the total drug dose is a simple way to prevent too high a toxicity for a given treatment. A bound on total dose may also represent a budget limit for expensive drugs [84].

However, fixed bounds on drug doses are not dynamic, i.e., they do not take into account specificities of the patient's metabolism and response to the treatment, other than by adapting daily doses to fixed coarse parameters such as body surface or weight (as is most often the case in the clinic so far). In order to get closer to actual toxicity limits, and hoping for a better result, it is possible to consider instead a lower bound on the number of healthy cells, as in [15]. In the same way, using a Malthusian growth model, where growth exponents are the targets of control, such a constraint becomes a lower bound on the asymptotic growth rate of the healthy cell population [138].

In the same way, a drug used in a treatment must reach a minimal concentration at the level of its target (which blood levels reflect only very indirectly) to produce therapeutic effects. Classically, clinical pharmacologists are accustomed to appreciating such efficacy levels by lower threshold blood levels, that are themselves estimated as functions of pharmacokinetic parameters such as 
first and second half-life times and distribution volume of the drug, with confidence interval estimates for a general population of patients. As in the case of toxicity, a more dynamic view is possible, by considering drug levels that decrease the number of cancer cells, that is, which yield a negative growth rate in the cancer cell population.

This leads to the definition of admissible sets for drug infusion flows, the union of $\{0\}$ and of a therapeutic range containing the infusion levels that are at the same time efficient and not too toxic (such a constraint is considered in [136]). Those admissible sets are rather difficult to take into account, however, as they lead to complex combinatorial problems.

An approach that is consequently often chosen (see [84] for instance) is to forget this constraint in the model and to a posteriori check that the optimal drug infusion schedules found are high enough to be efficient when they are nonzero.

That may be an elementary reason why so called bang-bang controls (i.e., all-or-none) are of major interest in chemotherapy optimisation: they are defined as controls such that at each time, either the drug infusion flow is the smallest possible (i.e., 0), or it is the highest possible. Even though it is now easy to use in the clinic (and also in ambulatory conditions) programmable pumps that may deliver drug flows according to any predefined schedule with long-lasting autonomy, solutions to optimisation problems often turn out to be bang-bang (tap open-tap closed).

But solutions to optimisation problems in cancer chemotherapy are not always bang-bang, when considerations other than on simple parallel growth of the two populations are taken into account, and this includes competition, when the two populations are in contact, e.g. in the bone marrow normal haematopoietic and leukaemic cells, or when both populations are submitted to a common - but differently exerted - physiological control, such as by circadian clocks [15].

Another interesting approach, relying on two models, one of them including the cell division cycle [114], and putting the optimal control problem with toxicity constraints, is developed in [54]. The optimal control problem is solved by using the industrial software gPROMS ${ }^{\circledR}$.

\section{Drug resistance}

Whereas therapeutic efficacy and limitation of toxic adverse effects are the first concern when dealing with chemotherapy, the frequent development of drug resistances in the target cancer cell populations is certainly the second bigger issue in the clinic. The development of such resistances may come from overexpression in individual cells of defence mechanisms as an exaggeration of physiological phenomena, such as are ABC transporters (the P-gp, or Pglycoprotein, being its most known representant), but they may also result, at least as likely, in proliferative populations encompassing mitoses, from mutations yielding more fit, i.e., resistant in the presence of drug, subpopulations. 
A classical solution to this problem is to forbid too low drug concentrations, that are supposed to create environmental conditions favourable to the development of more fit drug resistant cell populations without killing them, as is also the case, for instance, in antibiotherapy with bacteria. Nevertheless, other, more recent, arguments to support an opposite view, have been put forth: assuming that there exists a resistant cell population at the beginning of the treatment, or that it may emerge during the treatment, then delivering high drug doses often produces the effect to kill all sensitive cells, giving a comparative fitness advantage to resistant cells, that subsequently become very hard to eradicate. Thus a paradoxical solution has been proposed, at least in slowly developing cancers: killing just enough cancer cells to limit tumour growth, but letting enough of these drug sensitive cancer cells to oppose by competition for space the thriving of resistant cells, that are supposed to be less fit, but just the same, usually slowly, will invade all the tumour territory if no opponents are present [58,61]. Indeed, such free space left for resistant tumour cells to thrive, when high drug doses have been administered with the naive hope to eradicate all cancer cells, may result in the rise of tumours that escape all known therapeutics, a nightmare for physicians which is unfortunately too often a clinical reality. Hence the proposed strategy to avoid high doses, that are able to kill all sensitive cells, and to only contain tumour growth by keeping alive a minimal population of drug-sensitive tumour cells.

Both those constraints, toxicity and resistance, can be considered as part of the objective function by setting the objective to be a balance between two objectives. For instance, Kimmel and Swierniak in [75] proposed to minimise a linear combination of the number of cancer cells and of the total drug dose. This yields an unconstrained optimisation problem, that has a simpler resolution, while still taking into account the diverging goals of minimising the number of cancer cells and keeping the number of healthy cells high enough.

But whereas cancer and healthy cells are two quite distinct populations, with growth models that may easily be distinguished and experimentally identified by their parameters, it is more difficult to take into account the evolutionary lability (i.e., the genomic instability) and heterogeneity of cancer cell populations with respect to mutation-selection towards drug resistance, according to evolution mechanisms that are not completely elicited. Note that acquired (as opposed to intrinsic, i.e., genetically constitutive) drug resistance may result as well from individual cell adaptation (enhancement of physiological mechanisms) as from genetic mutations, both under the pressure of a drug-enriched environment, as discussed in [41]. In this respect, acquired resistance may be reversible, if no mutation has initiated the mechanism, or irreversible, and it is likely irreversible in the case of intrinsic resistance.

Ideally, the optimal solution of a therapeutic control problem should take into account both the drug resistance (using evolutionary cell population dynamics) and the toxicity constraints, but these constraints have usually been treated separately so far. Whereas the difficult problem of drug resistance 
control is certainly one of our concerns in a cell Darwinian perspective, in the sequel we shall present only results for the (easier) toxicity control problem.

\section{Identification of parameters: the target model and drug effects}

\subsection{Methods of parameter identification}

In the many works dedicated to modelling pharmacological control of tumour growth and its optimisation that have been published in the last forty years, when the issue of confronting a theoretical optimisation method with actual data has been tackled, quite different attitudes have been displayed. When identifying parameters of a biological model, one may use different methods, according to the nature of considered experimental data, their precision and reliability, and, also of course according to the scientific background of people in charge of identification. One may distinguish between at least three types of methods, all of which, to yield the best estimation of the parameters at stake usually rely on least squares minimisation, otherwise said minimisation of a $L^{2}$ distance between experimental quantitative observations and numerical features of the model, either direct outputs, such as, e.g., cell numbers, or computed statistical parameters, such as, e.g., mean cell cycle times.

\section{Probabilistic methods}

The first method is based on the theory of parameter estimation in statistical models, and supposes that a probability measure, depending on a set of statistical parameters, e.g., mean and variance of a probability density function (p.d.f.), is a priori given in a space of constitutive parameters of the model, e.g., coefficients in a set of differential equations. In its simplest form, estimation will result from the minimisation of the $L^{2}$ distance between a model p.d.f. and ancorresponding observed histogram, yielding with precision a best set of parameters for the p.d.f.. It may also result from more elaborated principles, such as maximum likelihood estimation (including the use of computational algorithms of the Expectation-Maximisation (EM) type, with or without the assumption of an underlying Markov chain), see the statistical literature on the subject, e.g., $[86,137]$ for a general presentation. (To this class of methods may also be related attempts to characterise by its statistical properties a chaotic deterministic system, as studied for instance in [82], when no actual model is given of the system, which is only supposed to have trajectories converging towards a chaotic attractor - on which they are dense -, an attractor which by definition is endowed with an invariant ergodic measure.) 
Optimisation of cancer drug treatments using cell population dynamics

\section{Control science and dynamical systems}

The second method comes from signal processing and control science. It is applied to the representation of dynamic systems by state-space models and it relies on the fact that the system is given by a set of ordinary differential equations, which may be converted by Laplace transform to the study of transfer functions, i.e., the system in the frequency domain. Such systems may be studied by their responses to input excitations to better characterise them. Presentations of such identification methods may be found in [93, 137].

\section{Inverse problems}

The third method, inverse problem solving, belongs to the domain of partial differential equations (PDEs). The models under consideration are close to the physical world and the method can comprise almost all situations, but requires specific studies for each case and nontrivial mathematics. The general principle is that observations of the real system represented by a PDE model correspond to an ill-posed problem, i.e., that the system of PDEs as it is given cannot be identified in a unique manner from the observations. Nevertheless, small regularisations (such as Tikhonov's), i.e., small modifications of the underlying differential operator, make the problem well-posed, i.e., amenable to the identification of its parameters in a unique manner. For a general presentation, see [76]. Recent developments on physiologically structured models may also be found in $[50,51,52,64,120]$.

\subsection{Parameters in macroscopic models of tumour growth}

In macroscopic models of tumour growth, parameter identification most often relies on imagery techniques, mainly radiological or MRI, as in [131] for brain tumour growth. But it is also possible to obtain tumour growth curves representing three-dimensional growth by using a method which may seem very coarse, but which has not found any really better competitor so far. It consists in growing a tumour (homograft or xenograft, i.e., of the same animal species, or of another) under the skin or on the skin of a laboratory rodent and measuring everyday by using a caliper diameters in three dimensions (one longitudinal and two orthogonal transverse) of the tumour, which is protected by the skin coating when the tumour is subcutaneous. It is possible only when the tumour is already palpable under the skin (or visible when it is on the skin), which excludes avascular tumours and generally involves histologically heterogeneous, but physically (i.e., in density with respect to water) homogeneous tumours. This allows an approximate estimation of the tumour mass, assumed to be proportional to the number of tumour cells, from its approximate volume and keeps the animal alive (until tumour weight reaches $10 \%$ of the animal body weight, at which point the animal is sacrificed for obvious ethical reasons). Coarse as it may seem, this method is still 
widely used because of its simplicity, see e.g., [35, 61, 124]. In a macroscopic (whole body) perspective, it should in principle also be possible to relate tumour growth with blood concentrations of biomarkers such as ACE, CA19.9 or PSA, known to be elevated in cancer or even only in tissue hyperplasia but they are not very specific; even for more specific antigenic biomarkers, models relating these quantities to actual cell population increase, to our knowledge, are still wanted. A recent review on this topic may be found in [113].

\subsection{Parameters in cell and molecular-based models of tissue growth}

At the cell population level, more easily quantifiable data have been recorded to identify model parameters in structured PDE models. They are linked to cell population samples, either with observation on a global population, such as given by flow cytometry (or FACS, for fluorescence activated cell sorting) $[16,17,18,19,20,95,135]$, or by observations on individual cells and statistics performed on the sampled population of individual cells marked with fluorescent proteins, such as FUCCI [125] in [25, 26]. These methods require previous cell staining, e.g. with propidium iodide (for flow cytometry), or hybridisation of intracellular proteins with external fluorescent proteins (for FUCCI). In all cases, in the presence of a stationary (i.e., asymptotic) distribution of cells may constitute the basis for applying an inverse problem method, as shown in $[12,51]$. A difference between these two sorts of experimental data (direct cell population or reconstruction of population with previous individual cell recordings) is that flow cytometry is a snapshot on a population of cells that are destroyed by the sorting process, whereas FUCCI investigates individual living cells without destroying them, a richer experimental situation.

\subsection{Measurement of pharmacodynamic effects}

Evaluation of the effects of a treatment using an anticancer drug involves measurements with and without treatment, which obviously is not ethically possible in most clinical situations. Nevertheless, in the case of low grade gliomas $[132,133]$, where tumours can evolve very slowly during many years, and for which it is known that no actually efficient therapy exists (in particular neurosurgery may be more detrimental than beneficial to the patient) therapeutists may unfortunately, but non unethically, find themselves in a situation of mere observers. In this case, macroscopic images of tumours may be used, providing parameter estimation without treatment, which may then serve as a basis for comparisons with treated gliomas. The observations are always radiological or MRI. But in cell cultures and in animals, such experimental observations are of course much easier and may allow comparing parameters of interest evaluated by using any of the methods mentioned above in different situations: cell cultures, fresh blood samples from patients, tumour growth curves $[11,18,19,20,35,117]$ and it is then possible to propose optimised treatments based on these estimations $[10,15,35]$. 
Optimisation of cancer drug treatments using cell population dynamics

\section{Therapeutic optimisation procedures}

In the previous sections we have described the drugs used in chemotherapy, various cell population dynamic models and the objectives and constraints considered in chemotherapy optimisation problems that have been published. Those three topics can be seen as components of an optimisation model. In order to get quantitative results, the parameters of this model should be estimated by using the techniques presented in Section 5 . Then, one has to choose an optimisation procedure to solve the optimisation problem considered.

When choosing an optimisation procedure, one first needs to identify what are the optimisation variables. For chemotherapy optimisation, there are two main situations: either the optimisation variables are some parameters of a predefined infusion scheme or they are the infusion scheme itself, represented by a time-dependent control function $u(t)$ (cf. Section 4.1).

\subsection{Graphic optimisation}

"Graphic optimisation" simply consists in plotting the value of the objective for all admissible points. It is a very simple scheme and the only requirement for its success is that the admissible set must have a nonempty interior. It also provides graphics to present the result.

Graphic optimisation suits particularly the case of a predefined infusion scheme with only a few parameters. For instance, this technique was used by Webb [138] and Panetta and Adam [114]. These authors considered models

of the McKendrick type (see Section 3) and they searched for the best period of periodic drug infusions, i.e., the period of predefined drug infusion schemes that minimises the growth rate of cancer cells. Altinok et al. [6] proposed a cellular automaton model controlled by two predefined infusion schedules of drugs where the parameter is the phase difference between a circadian clock and the drug infusion.

The drawback of this method is that when the number of parameters grow, the time necessary for the resolution of the problem grows exponentially. Moreover, graphics are less practical when the dimension exceeds 3 . The classical solution is, rather, to make use of a more evolved optimisation algorithm. However, if one needs an evolved optimisation algorithm anyway, one might as well consider an optimal control problem, in which the whole infusion schedule is the optimisation variable.

\subsection{Pontryagin's maximum principle}

An optimal control problem is an optimisation problem where the objective is a function of the state variables $x(\cdot): \mathbb{R}_{+} \rightarrow \mathbb{R}^{n}$ of a dynamical system and of the controlled variables $u(\cdot): \mathbb{R}_{+} \rightarrow \mathbb{R}^{m}$ that control the dynamical system. It can be written formally as 


$$
\begin{array}{r}
\min _{u(\cdot), T} \int_{0}^{T} f^{0}(t, x(t), u(t)) d t+g^{0}(T, x(T)) \\
\dot{x}(t)=f(t, x(t), u(t)), \quad \forall t \in[0, T] \\
u(t) \in U_{t}, \quad \forall t \in[0, T] \\
x(0)=x_{0}, \quad x(T) \in M_{1}
\end{array}
$$

Here, $f^{0}$ is the cost function and $g^{0}$ is the final cost. The final time $T$ can be either fixed or be part of the control. The dynamical system is represented by function $f$, which gives the evolution of $x$ and is controlled by $u$. At each time, the control may be subject to constraints represented by the set $U_{t}$ and the set $M_{1}$ is a subset of $\mathbb{R}^{n}$ representing conditions on the final state. If we replace the constraints $x(0)=x_{0}$ and $x(T) \in M_{1}$ by $x(T)=x(0)$, we have a $T$-periodic optimal control problem (see [28] for more precision on the consequences of this model).

A major tool of optimal control is Pontryagin's maximum principle [121]. It gives necessary optimality conditions for the optimal trajectories. We denote the Hamiltonian of the system by

$$
\left.H\left(t, x, p, p^{0}, u\right)=\sum_{i=1}^{n} p^{i} f_{i}(t, x, u)\right\rangle+p^{0} f^{0}(t, x, u),
$$

where $p=\left(p^{1}, \ldots, p^{n}\right) \in \mathbb{R}^{n}$ and $p^{0} \in \mathbb{R}$. If $u(\cdot)$ associated to the trajectory $x(\cdot)$ is an optimal control on $[0, T]$, then there exists a continuous application $p(\cdot)$ called the adjoint vector and a nonpositive number $p^{0}$ such that for almost all $t \in[0, T]$,

$$
\dot{x}(t)=\frac{\partial H}{\partial p}\left(t, x(t), p(t), p^{0}, u(t)\right), \quad \dot{p}(t)=-\frac{\partial H}{\partial x}\left(t, x(t), p(t), p^{0}, u(t)\right)
$$

and we have the maximisation condition for almost all $t \in[0, T]$,

$$
H\left(t, x(t), p(t), p^{0}, u(t)\right)=\max _{v \in U_{t}} H\left(t, x(t), p(t), p^{0}, v\right) .
$$

If in addition, the final time to reach the target $M_{1}$ is not fixed, we have the condition

$$
\max _{v \in U_{T}} H\left(T, x(T), p(T), p^{0}, v\right)=-p^{0} \frac{\partial g^{0}}{\partial t}(T, x(T))
$$

and if $M_{1}$ is manifold of $\mathbb{R}^{n}$ with a tangent space $\mathrm{T}_{x} M_{1}$ at $x$, we have

$$
p(T)-p^{0} \frac{\partial g}{\partial t}(T, x(T)) \perp \mathrm{T}_{x(T)} M_{1} .
$$

Under the conditions of the Pontryagin's maximum principle given in Equations (17), (18), we have

$$
\frac{d}{d t} H\left(t, x(t), p(t), p^{0}, u(t)\right)=\frac{\partial H}{\partial t}\left(t, x(t), p(t), p^{0}, u(t)\right)
$$


and thus if $f, f^{0}$ and $U_{t}$ do not depend on $t$, then $H$ does not depend on $t$ and $\max _{v \in U_{t}} H\left(t, x(t), p(t), p^{0}, v\right)$ is constant.

Thanks to Pontryagin's maximum principle one is often able to determine the optimal control as a function of the adjoint vector. Nevertheless, the adjoint vector is not easy to compute. It is defined through its value at the terminal point, $p(T)$, and solutions to the associated boundary value problem are difficult to compute, nor need they be unique.

For Pontryagin's maximum principle to be applicable, the cell population model must be a set of ODEs controlled by drug infusions, as presented in Section 3.1. The authors generally minimise the number of cancer cells at final time with a bound on the instantaneous drug flow. The total dose is either constrained to be bounded or is part of the objective, a smaller dose improving the objective. When the information provided by Pontryagin's maximum principle is enough to know the optimal control, as in [49, 56, 75, 84], it gives the control, i.e., the solution to the problem, in an explicit formula, without any discretisation. This is then a very valuable information. Unfortunately, for most optimal control problems, and optimal control arising from chemotherapy problems are not an exception, we do not have enough information and we have to use a numerical algorithm to solve the problem.

\subsection{Numerical methods for optimal control problems}

Two classes of numerical methods exist for optimal control problems, namely indirect methods, also called shooting methods, and direct methods.

\section{Shooting method}

The shooting method is based on the observation that, if ever we knew the value $p_{0}=\left(p_{0}^{0}, p_{0}^{1}, \ldots, p_{0}^{n}\right)$ of the adjoint state at the initial point, we could get the optimal controls time by time. Thus we define the shooting function $G\left(p_{0}\right)$ such that $G\left(p_{0}\right)=0$ if and only if $p(T)$ satisfies the final conditions (recall that $T$ is the final time). The shooting method simply consists in solving the equation $G\left(p_{0}\right)=0$, with variable $p_{0}$, for instance by a Newton method.

A variant of the shooting method was used in [84] for chemotherapy optimisation. Ledzewicz et al. considered two drugs that act on a Gompertzian model: one is an anti-angiogenic, which controls the carrying capacity of the tumour and the other is a cytotoxic drug, which controls a death term. The pharmacodynamics of the drugs was modelled by linear differential equations. The authors apply Pontryagin's maximum principle on this model with the objective of minimising the quantity of tumour cells and as constraints an upper bound on the drug instantaneous flow and an upper bound on drug total dose. The authors obtained the optimal control as a function of the adjoint vector. The optimal control reaches the dose bound when a function related to the adjoint is nonzero and it follows a singular curve when this function vanishesd along an interval. They then used a shooting method to construct the 
optimal control as a feedback function from these adjoint-vector dependent singular curves. Algorithmic details are given in [99].

In general, shooting methods give very precise results but the structure of commutations, given by studying Pontryagin's maximum principle, must be known in advance for them to be efficient. When this structure is unknown, one can still perform direct methods, which we describe next.

\section{Direct methods}

Direct methods consist of a total discretisation of the control problem and then of solving the finite dimensional optimisation problem obtained. The discretisation of an optimal control problem results in an optimisation problem with a large number of variables. The theory of differentiable optimisation is the classical tool for such problems [24, 29, 111]. However, in order to overcome the limits of differentiable optimisation, some authors use stochastic algorithms to solve the discretised problem. We next give some examples of these techniques in the context of chemotherapy.

\section{Gradient algorithm}

When the problem is formulated without any state constraint, one can use the gradient algorithm, as in [118]. The authors proposed a cell-cycle dependent model written with one ODE by cell-cycle phase. They controlled the transition and death rates and optimised a linear combination of the number of cancer cells and of the total dose of drugs. The gradient algorithm starts here with an initial control strategy $u_{0}$ and the associated trajectory $x_{u_{0}}$. It consists in successive improvements of the discretised objective $F^{0}(u)=\sum_{l=0}^{N} f^{0}\left(t_{l}, x_{u}\left(t_{l}\right), u\left(t_{l}\right)\right)+g^{0}\left(T, x_{u}(T)\right)$ by

$$
u_{k+1}=P_{U}\left(u_{k}-\alpha \nabla F^{0}\left(u_{k}\right)\right)
$$

where $U$ is the set of admissible controls and $\alpha$ is a length step chosen in order to guarantee a sufficient decrease of the objective, for instance with an Armijo or Wolfe line search rule. When computing the gradient of the objective with respect to the control, there appears an adjoint vector which is a discrete version of the adjoint vector in Pontryagin's maximum principle.

\section{Uzawa algorithm}

An optimal control problem with $K$ constraints is a problem of the form of problem (16) where we add constraints $\int_{0}^{T} f^{i}(t, x(t), u(t)) d t+g^{i}(T, x(T)) \leq 0$ for $i=1, \ldots, K$. For such problems, direct methods are particularly suited and the discretised optimal control problem can be solved by the Uzawa algorithm.

We denote $F^{i}(u)=\sum_{l=0}^{N} f^{i}\left(t_{l}, x_{u}\left(t_{l}\right), u\left(t_{l}\right)\right)+g^{i}\left(T, x_{u}(T)\right)$ and we introduce the Lagrangian 


$$
L(u, \lambda)=F^{0}(u)+\sum_{i=1}^{K} \lambda^{i} F^{i}(u)
$$

where $\lambda$ is a vector with one coordinate by state constraint called a Lagrange multiplier. At a given iterate $\left(u_{k}, \lambda_{k}\right)$, we solve

$$
u_{k+1}=\arg \min _{u} L\left(u, \lambda_{k}\right)
$$

by a nonconstrained optimisation algorithm, as is the gradient algorithm, and then we compute

$$
\lambda_{k+1}^{i}=\max \left(0, \lambda_{k}^{i}+\alpha F^{i}\left(u_{k+1}\right)\right), \quad \forall i \in\{1, \ldots, K\} .
$$

where $\alpha$ is an appropriate step size. If the constraint is an equality constraint instead of an inequality constraint, we accept nonpositive values for $\lambda$ and we do not perform the maximum against 0 .

Basdevant et al. used the Uzawa algorithm in [15] to solve the problem of minimising the number of cancer cells while maintaining the number of healthy cells over a tolerability threshold. They modelled the cell population dynamics and the action of the drug by a set of coupled differential equations.

In [26], we solved the problem of minimising the asymptotic growth rate of the cancer cell population while keeping the asymptotic growth rate of the healthy cell population over a prescribed threshold; see a sketch of the method and of its results below in Section 7. We modelled the cell population dynamics by a McKendrick model physiologically controlled by a circadian clock, considering a phase-dependent drug acting on transitions. We firstly discretised the problem and then solved it by using a Uzawa algorithm with augmented Lagrangian. That is to say, we replaced the Lagrangian by

$$
L_{c}(u, \lambda)=F^{0}(u)+\frac{1}{2 c} \sum_{i=1}^{K}\left(\max \left(0, \lambda^{i}+c F^{i}(u)\right)^{2}-\left(\lambda^{i}\right)^{2}\right) .
$$

Compared to the classical Lagrangian, the augmented Lagrangian has better convergence and stability properties for a small computational cost.

Other differentiable optimisation algorithms may also be used, depending on the properties of the problem at stake. In general, all these algorithms give a local optimal solution quickly but they do not give any guarantee that the control solution produced is a global optimum. In order to overcome this drawback, some authors chose to use stochastic algorithms instead.

\section{Stochastic algorithms}

Stochastic algorithms are algorithms that use a random number generator to find the optimal solutions of a given problem. These random numbers are used to explore the admissible control set with the hope that the optimal controls will eventually be hit. Each stochastic optimisation algorithm is a compromise 
between focusing on good solutions and letting enough freedom to exploration in order not to miss the global optimum. See $[68,129]$ for more details on this subject.

In [1], Agur et al. considered an age-structured cell cycle model with deterministic cycle phase lengths. The drug under consideration is toxic for cells in one of the phases only. They considered a composite objective function that takes into account the number of cancer and healthy cells in the end and a measure of the survival of the patient. They assumed that a patient survives if at no time the number of healthy cells falls below a threshold. The authors compared three versions of simulated annealing. They first defined the neighbourhood of every point of the admissible set, that is, at every point, they defined the possible ways to go to another point. This neighbourhood should be large enough to give freedom to the algorithm but not too large because otherwise the computational cost of searching the neighbourhood would be dissuasive. Then simulated annealing gives the rule for the acceptance or rejection of a neighbour, which gets stricter when a parameter, call the temperature, decreases. In theory, if the temperature is decreased properly, the iterates converge to an optimum of the problem. In practice, convergence may be desperately slow. The other two heuristics presented in the paper do the same work but with simplified rules, that do not guarantee convergence to an optimum but have smaller computational costs.

Villasana et al. proposed in [136] an ODE model with three types of cells: cancer cells in interphase (i.e., $G_{1}, S$ and $G_{2}$ ), cancer cells in mitosis phase $(M)$ and healthy cells. Each type of cells has a particular dynamic and there are interactions between them. They considered a combination of a cytotoxic and of a cytostatic drug and they wanted to minimise the number of cancer cells while keeping the number of healthy cells above a threshold. They used the covariance matrix adaptation evolutionary strategy (CMA-ES) to solve this problem. This is an algorithm based on probabilistic mutations of the current iterates and on a selection of the best ones [68]. The covariance matrix adaptation is a way to give the mutations directions for them to be more effective.

\section{Focus: cancer therapeutics to control long-term cell population behaviour in structured cell population models}

\subsection{Linear and nonlinear models}

We have presented in $[25,26]$ a method based on the control of eigenvalues in an age-structured model, yielding the numerical solution of an optimal control problem in the context of cancer chronotherapeutics, and we sum up below some of its results as regards system modelling, identification, and theoretical therapeutic optimisation. To this goal, we used an age-structured 
cell population model, since our aim was to represent the action of cytotoxic anticancer drugs, which always act onto the cell division cycle in a proliferating cell population. The model chosen, of the McKendrick type [102], is linear. This may be considered as a harsh simplification to describe biological reality, which involves nonlinear feedbacks to represent actual growth conditions such as population size limitation due to space scarcity. Nonetheless, having in mind that linear models in biology are just linearisations of more complex models (for instance considering the fact a first course of chemotherapy will most often kill enough cells to make room for a non space-limited cell population to thrive in the beginning) we think that it is worth studying population growth and its asymptotic behaviour in linear conditions and thus analyse it using its growth (or Malthus) exponent. This first eigenvalue of the linear system may be considered as governing the asymptotic behaviour, at each point where it has been linearised, of a more complex nonlinear system, as described in $[21,22]$.

\subsection{Age-structured models for tissue proliferation and its control}

We know that circadian clocks [87, 88, 89, 90, 91, 92] normally control cell proliferation, by gating at checkpoints between cell cycle phases (i.e., by letting cells pass to the next phase only conditionally). We also know that circadian clock disruption has been reported to be a possible cause of lack of physiologically control on tissue proliferation in cancer [91], a fact that we will represent in our model to distinguish between cancer and healthy cell populations.

The representation of the dynamics of the division cycle in proliferating cell proliferations by physiologically structured partial differential equations (PDEs) is thus a natural frame to model proliferation in cell populations, healthy or tumour. The inclusion in such proliferation models of targets for its control, physiological (circadian) and pharmacological (by drugs supposed to act directly on checkpoints), allows to develop mathematical methods of their analysis and therapeutic control [25, 26, 36], in particular for cancer chronotherapeutics, i.e., when the drug control is made $24 \mathrm{~h}$-periodic to take advantage of favourable circadian times.

Physiologically structured cell population dynamics models have been extensively studied in the last 20 years, see Section 3.4 for some examples. We consider here typically age-structured cell cycle models, in which the cell division cycle is divided into $I$ phases (classically $4: G_{1}, S, G_{2}$ and $M$ ), and the variables are the densities $n_{i}(t, x)$ of cells having age $x$ at time $t$ in phase $i$. Equations read

$$
\left\{\begin{array}{l}
\frac{\partial n_{i}(t, x)}{\partial t}+\frac{\partial n_{i}(t, x)}{\partial x}+d_{i}(t, x) n_{i}(t, x)+K_{i \rightarrow i+1}(t, x) n_{i}(t, x)=0 \\
n_{i+1}(t, 0)=\int_{0}^{\infty} K_{i \rightarrow i+1}(t, x) n_{i}(t, x) d x \\
n_{1}(t, 0)=2 \int_{0}^{\infty} K_{I \rightarrow 1}(t, x) n_{I}(t, x) d x
\end{array}\right.
$$


together with an initial condition $\left(n_{i}(t=0, .)\right)_{1 \leq i \leq I}$. This model was first introduced in [42] and further studied in other publications, among which $[39,40]$. In this model, in each phase $i$, cells are ageing with constant speed 1 (transport term), they die with rate $d_{i}$ or with rate $K_{i \rightarrow i+1}$ go to next phase, in which they start with age 0 . To represent the effect of circadian clocks on phase transitions [91], one may consider time-periodic coefficients $d_{i}$ and $K_{i \rightarrow i+1}$, the period being in this case 24 hours.

\subsection{Basic facts about age-structured linear models}

One of the most important facts about linear models is the trend of their solutions to exponential growth. The study of the growth exponent, first eigenvalue of the system, is therefore crucial. Solutions to sytem (19) satisfy (if the coefficients are time-periodic, or stationary) $n_{i}(t, x) \sim C^{0} N_{i}(t, x) e^{\lambda t}[119]$, where $N_{i}$ are defined by

$$
\left\{\begin{array}{l}
\frac{\partial N_{i}(t, x)}{\partial t}+\frac{\partial N_{i}(t, x)}{\partial x}+\left(\lambda+d_{i}(t, x)+K_{i \rightarrow i+1}(t, x)\right) N_{i}(t, x)=0, \\
N_{i+1}(t, 0)=\int_{0}^{\infty} K_{i \rightarrow i+1}(t, x) N_{i}(t, x) d x \\
N_{1}(t, 0)=2 \int_{0}^{\infty} K_{I \rightarrow 1}(t, x) N_{I}(t, x) d x \\
N_{i}>0, \quad N_{i}(t+T, .)=N_{i}(t, .), \quad \sum_{i} \int_{0}^{T} \int_{0}^{\infty} N_{i}(t, x) d x d t=1
\end{array}\right.
$$

with $T$-periodic coefficients.

We focus now on the case of stationary phase transition coefficients $\left(K_{i \rightarrow i+1}(t, x)=K_{i \rightarrow i+1}(x)\right)$ and we do not consider death rates $\left(d_{i}=0\right)$. Note that if one considers constant nonzero death rates, the problem does not change, only the eigenvalue $\lambda$ is then in fact $\lambda+d$, as one can see in the equations of system (20). As shown in [42], the first eigenvalue $\lambda$ is then solution of the following equation, which in population dynamics is referred to, in the 1-phase case $(I=1)$ with no death term, as Euler-Lotka's equation

$$
\frac{1}{2}=\prod_{i=1}^{I} \int_{0}^{+\infty} K_{i \rightarrow i+1}(x) e^{-\int_{0}^{x} K_{i \rightarrow i+1}(\xi) d \xi} e^{-\lambda x} d x .
$$

Integrating the first equation of System (19) along its characteristics [119], we can in the stationary case with no death rate derive the formula

$$
n_{i}(t+x, x)=n_{i}(t, 0) e^{-\int_{0}^{x} K_{i \rightarrow i+1}(\xi) d \xi} .
$$

This can be interpreted in the following way: the probability for a cell which entered phase $i$ at time $t$ to stay for at least an age duration $x$ in phase $i$ is given by 


$$
P\left(\tau_{i} \geq x\right)=e^{-\int_{0}^{x} K_{i \rightarrow i+1}(\xi) d \xi} .
$$

The time $\tau_{i}$ spent in phase $i$ is thus a random variable on $\mathbb{R}_{+}$, with probability density function $f_{i}$ given by

$$
d P_{\tau_{i}}(x)=f_{i}(x) d x=K_{i \rightarrow i+1}(x) \cdot e^{-\int_{0}^{x} K_{i \rightarrow i+1}(\xi) d \xi} d x,
$$

or equivalently:

$$
K_{i \rightarrow i+1}(x)=\frac{f_{i}(x)}{1-\int_{0}^{x} f_{i}(\xi) d \xi}
$$

\subsection{FUCCI (cell cycle) reporters to identify model parameters}

FUCCI (for fluorescence ubiquitination-based cell cycle indicator) is a recent cell imaging technique that allows tracking progression within the cell cycle of an individual cell [125] after hybridisation with fluorescent proteins of cell cycle phase characteristic indicators (geminin and Cdt1) involved in the ubiquitination, i.e., natural degradation, of the actual proteic determinants of evolution in the cell cycle. We used FUCCI data, that consisted in time series of the intensity of red and green fluorescence emitted by individual NIH 3T3 cells (mouse embryonic fibroblasts) within an in vitro homogeneous population proliferating without any control in a liquid medium. This allowed us to measure the time an individual cell spent in the $G_{1}$ phase and in the phases $S / G_{2} / M$ of the cell cycle. Our data consisted of cell cycle phase durations from 55 proliferating cells. Note that is fully justified in our case to assume $K_{i \rightarrow i+1}(t, x)=K_{i \rightarrow i+1}(x)$ since these experimental conditions correspond to cells proliferating in a completely independent manner, without any communication nor external control on their proliferation. It is noteworthy that, though we deal with PDEs, our method is simpler than methods involving inverse problem solving (which nevertheless have been used on comparable situations, for instance in [51]), and this is due to the fact that using the FUCCI reporter technique we have access to precisely defined data in individual cells, with the counterpart that quite few individual proliferating cells have been recorded. A graph representing a time series from an individual cell and the method used to record phase durations is presented on Figure 1.

We used these experimental data to identify the parameters of our model by fitting shifted Gamma distributions $f(x)=\rho^{-k}(\Gamma(k))^{-1}(x-a)^{k-1} e^{-\frac{(x-a)}{\rho}}$ on $\left[a,+\infty\right.$ [ to frequencies of appearance of $G_{1}$ and $S / G_{2} / M$ durations within the population (recall that variable $x$ stands for age in each one of the two phases). These Gamma distributions were approximations of the probability density functions of the random variable corresponding to the time spent in $G_{1}$ and $S / G_{2} / M$. This enabled us to determine the expression of the transitions rate according to the formula (22). We then compared the solutions of the system with cell recordings, that had previously been synchronised "by hand", i.e., all recordings were artificially made to start simultaneously at the beginning of $G_{1}$ phase. The result is shown on Figure 2. Note that using an 


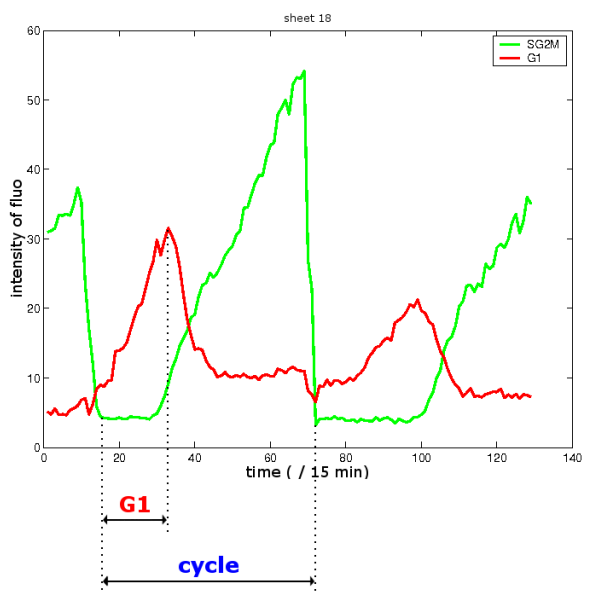

Fig. 1. Graphic method used to determine the durations of the whole cell cycle and of $G_{1}$ phase. The duration of phase $S / G_{2} / M$ was deduced by subtracting the duration of phase $G_{1}$ from the duration of the cell cycle.

inverse problem method - see Section 5 - instead of ours could have consisted here in determining the parameters of the model, i.e., $K_{i \rightarrow i+1}$ transition functions, by minimising a $L^{2}$ distance between this experimental data curve and a theoretical, parameter-dependent curve representing these data.

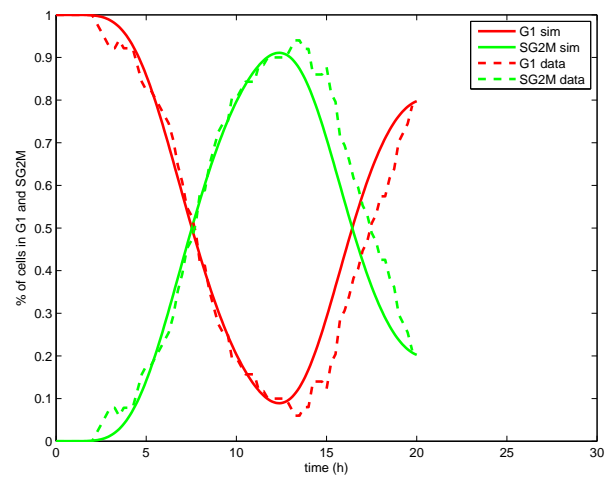

Fig. 2. Time evolution of the percentages of cells in the phases $G_{1}$ (red or deep grey) and $S / G_{2} / M$ (green or light grey) from biological data (dashed line) and from numerical simulations (solid line). Our model results in a reasonably good fit to biological data. 


\subsection{Optimising eigenvalues as objective and constraint functions}

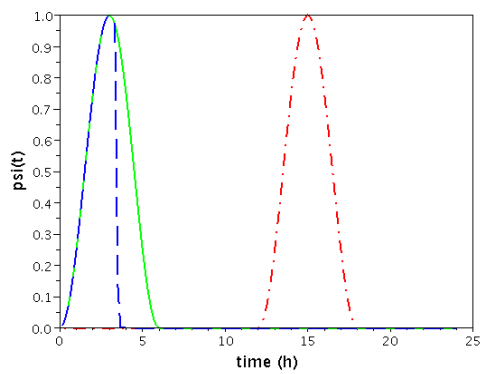

Fig. 3. Drug and circadian controls, healthy cell population case. Cosine-like functions modelling the drug and circadian controls for transition from $G_{1}$ to $S / G_{2} / M$ (dash-dotted line) and for transition from $S / G_{2} / M$ to $G_{1}$ in healthy cells. The "natural" (drug-free) control for $S / G_{2} / M$ to $G_{1}$ transition corresponds to the solid line, the drug-induced one to the dashed line.

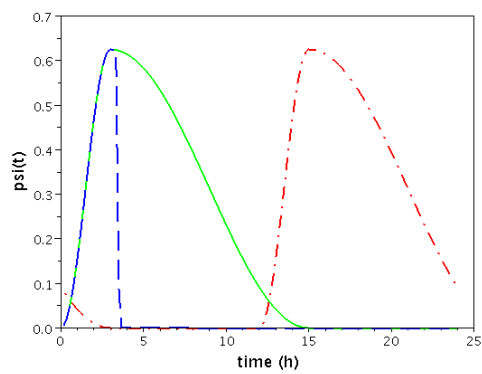

Fig. 4. Drug and circadian controls, cancer cell population case. Cosine-like functions modelling the drug and circadian controls for transition from $G_{1}$ to $S / G_{2} / M$ (dash-dotted line) and for transition from $S / G_{2} / M$ to $G_{1}$ in cancer cells. The "natural" (drug-free) control for $S / G_{2} / M$ to $G_{1}$ transition corresponds to the solid line, the drug-induced one to the dashed line.

We then used combined time-independent data on phase transition functions, obtained from experimental identification of the parameter functions $K_{i \rightarrow i+1}(t, x)=\kappa_{i}(x)$ in the uncontrolled model, with cosine-like functions representing the periodic control on these transitions by circadian clocks, together with free-running drug infusion regimens. The drug infusion regimens were optimised using a Uzawa method with an augmented Lagrangian (see Section 6.3 or [29] for algorithmical details), aiming at decreasing the growth rate in a cancer cell population (objective) while preserving the same in a healthy cell population (constraint) by maintaining it over a prescribed 
threshold. The idea is the same as in [15], except that we deal here with cell population growth exponents instead of cell numbers.

We considered two cell populations, that we called cancer cells and healthy cells. In these simulations, we took into account cell death via a constant death rate, the same for both populations. We made the two cell populations only differ by their circadian control function $\psi$ and we assumed that there was no interaction between the two populations, healthy and cancer. We took for this circadian control a continuous piecewise cosine-like function for each phase (Figure 3). We assumed that cancer cell populations still obey circadian control at these main checkpoints but less faithfully, and we modelled their behaviour by a looser answer to the circadian control signal (Figure 4).

Transitions from one phase to the other are described by the transition rates $K_{i \rightarrow i+1}(t, x)$. We took them with the form

$$
K_{i \rightarrow i+1}(t, x)=\kappa_{i}(x) \psi_{i}(t)\left(1-g_{i}(t)\right)
$$

where $\kappa(x)$ is the transition rate of the cell without circadian control identified from FUCCI data, $\psi_{i}(t)$ is the natural circadian control modelled by a cosinelike function and $g_{i}(t)$ is the effect at the cell level of the drug infusion at time $t$ on the transition rate from phase $i$ to phase $i+1$. No drug corresponds to $g_{i}(t)=0$, a transition-blocking infusion corresponds to $g_{i}(t)=1$. We assumed that the drug has the same effect on both populations, which couples their behaviours through the drug infusions.

We solved the constrained optimisation problem $[25,26]$ with Uzawa algorithm. After convergence of the algorithm, we get the locally optimal strategy, shown in our case on Figure 5, defining on $[0 ; 24]$ the 24h-periodic function $g_{2}$ (assuming that $g_{1}=0$, that is here mimicking the action of an anticancer drug - as 5-fluorouracil - active in $S$ phase only). It can be seen that it mainly consists in forbidding transitions when healthy cells do no change phase, thus harming cancer cells only.

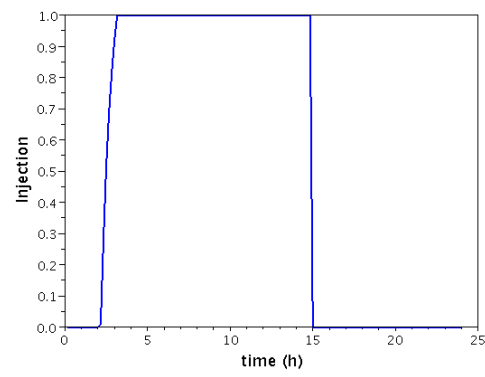

Fig. 5. Locally optimal periodic drug infusion strategy (function $g_{2}$, see text for details) found by the optimisation algorithm. 
The result of the optimised drug infusion regimen rates is shown on Figure 6 , where it can be seen that the asymptotic growth rate of cancer cells, initially positive and higher than the one of healthy cells, has been rendered negative by the periodic treatment exerted on transition rates while the new growth rate of healthy cells, though moderately affected by the treatment, remains positive.

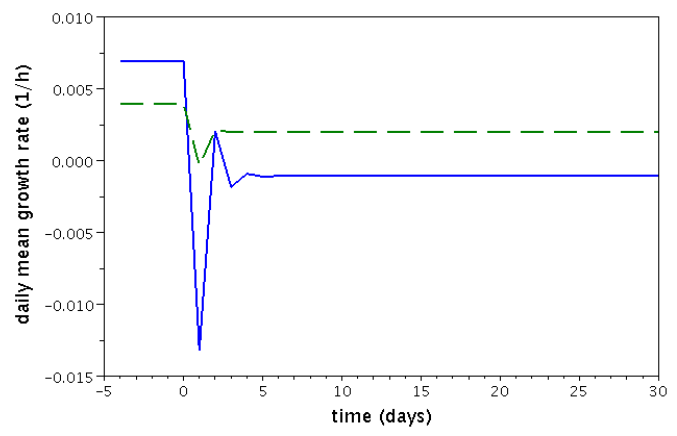

Fig. 6. Daily mean growth rates for cancer (solid line) and healthy cells (dashed line) when starting drug infusion at time 0 . After a 10-day transient phase, the drugforced biological system stabilizes towards the expected asymptotic growth rate

Note that the FUCCI technology only enables us to distinguish between cells in $G_{1}$ and $S / G_{2} / M$, without distinction between $S, G_{2}$ and $M$. However, we may note that the method used in [26] to identify phase transitions relies in fact on the probability distribution of durations of phases. Since the duration of the phase $M$ is known to be most of the time very short, with almost zero variability within cell populations, it would be legitimate to considered it as fixed, as 1 hour, say, and that the recorded variability of $S / G_{2} / M$ is in fact the variability of $S / G_{2}$. Thus, we could have considered that we were dealing in this identification process with a transition function from $S / G_{2}$ to $M$ instead of the one from $S / G_{2} / M$ to $G_{1}$. In this case, the transition function from $M$ to $G_{1}$ could have been modelled by an indicator function, representing the fact that mitotic cells divide and enter the $G_{1}$ phase once they are old enough. Under these assumptions, we could have applied our optimisation problem to an age-structured model accounting for 3 phases of the cell cycle, $G_{1}, S / G_{2}$ and $M$. As a first step in our analysis, we preferred keeping the model as simple as possible, in the absence of actual knowledge of the duration of $M$ phase, but taking it as fixed, for instance to 1 hour, such considerations might be developed in future works to assume that we have, thanks to FUCCI reporters, accessed the main two checkpoints, $G_{1} / S$ and $G_{2} / M$. 


\section{Future prospects: multitarget multidrug delivery optimisation in physiologically structured cell population models}

From a therapeutic point of view, we have only represented the action of one drug acting on cell cycle phase transitions (on only one transition, $G_{2} / M$, if it acts on $S$ phase). We know indeed that, in particular via p53, drugs that create DNA damage ultimately act by blocking the cell cycle at checkpoints, mainly $G_{1} / S$ and $G_{2} / M$, and this only subsequently sends the cell into apoptosis, therefore cell cycle transition checkpoints are the most accurate targets to represent the effect of most cytotoxic drugs. The action of spindle poisons, such as taxans and vinca alcaloids, that block the cell cycle in $M$ phase (but not at $G_{1} / S$ or $G_{2} / M$ ), might also be represented by using the same formalism.

Now, in everyday oncology, most treatments use combinations of drugs that exert their action in synergy on different targets. These drugs may act on phase transitions only, but they may also act by inhibition of growth factor receptors (such as cetuximab, or tyrosine kinase inhibitors), impinging on the speed $v_{1}$, depending on age $x$ at which $G_{1}$ phase is run through. In this case, one may use, as in [69], an extended version of model (19), where

$$
\frac{\partial}{\partial t} n_{1}(t, x)+\frac{\partial}{\partial x}\left(v_{1}(x) n_{1}(t, x)\right)+d_{1}(t, x) n_{1}(t, x)+K_{1 \rightarrow 2}(t, x) n_{1}(t, x)=0 .
$$

Another possibility would be to introduce a non proliferating, or quiescent, phase $G_{0}$ exchanging cells with $G_{1}$ and to represent the action of growth inhibitors by a control of these exchanges.

In Section 7, summing up [26], and following [15], we have focused on mathematical models of tissue growth having in mind only the problem of limiting drug toxicity to healthy cell populations to optimise cancer treatments. In the future, making available models of the emergence of drug-resistant cell subpopulations under drug pressure in a cell Darwinian perspective, we will simultaneously tackle at the cell population level the constraints of drug resistance in tumour cells and of toxicity to healthy tissues, to propose globally efficient combined therapies using at least two complementary drugs.

In a multiscale perspective, integrating a representation of the vasculature around a cancer cell population will also allow us to represent and optimise the action of combined therapies associating cytotoxic and antiangiogenic drugs, as in $[49,83]$.

To be relevant for actual clinical applications, models based on the representation of evolving structured populations will also need to be integrated in a whole-body level, from the infusion of drugs into the central compartment of general blood circulation until the actions they exert at the peripheral sites on proliferating cell populations. This has partly been done, but still without control, and in the case of an avascular tumour, in [31]. Moreover, to take into account in a dynamic way the constraint of limiting unwanted toxicity 
to healthy tissues, cell population growth models will have to separately represent both tumour and healthy cell populations, with therapeutic control, wanted or not, exerted on both, and possibly with competition between the two populations, as is the case for space in the bone marrow between leukaemic and normal haematopoietic cells at different stages of their maturation.

Whole-body integration of different spatial scales of description of pharmacological control on tumour and on healthy cell populations should certainly go as down as possible at the single cell level, including for instance nucleocytoplasmic transport to model the control by p53 of the cell cycle in case of damage to the DNA, as produced by cytotoxic drugs, and it must necessarily contain an intermediate tissue level as the main level of description, for cancer is certainly a tissue disease, which may be controlled only at the tissue, i.e., at the cell population level. Then higher levels of integration: whole-body, and for the individualisation of treatments (in particular adaptation of whole-body model parameters to clusters of patients), population of individuals, must be considered, as sketched in [38].

Apart from toxicity issues, at this intermediate level (the tissue), will also be considered in models presently under construction [41] the possible evolution of cells towards drug resistance, which is the other main problem encountered in cancer therapeutics. To prevent or overcome the emergence of resistant cell subpopulations, it is usually better to make use of several anticancer drugs acting on different targets, to avoid as much as possible strategies used by cancer cells, which, due to their genomic instability, easily adapt by mutations to single-drug therapies. This has been the case for instance with imatinib, a drug that has completely changed the prognosis of Chronic Myelogenous Leukaemia (CML), but has nevertheless, after being considered as a miracle drug, also be confronted to the issue of resistance in CML cells [126]. Future optimisation problems in cancer therapeutics will have to take into account as a constraint, given the possibility to induce drug resistance, to limit it, as much as possible, not necessarily by a complete eradication of tumour cells, but, as sketched in [58,61], more realistically by its containment, and this will likely more easily done by using combinations of therapies than by using monotherapies.

\section{Discussion and conclusion}

We have presented in this chapter firstly a brief review of models of cancer that have been used or may be used to tackle the general problem of therapeutic optimisation in oncology. As sketched elsewhere [37, 38], theoretic drug delivery optimisation is the last step of therapeutic optimisation, which must rely firstly on an accurate representation of the behaviour of targets (wanted and unwanted) without treatment and on the changes the means of action of the physician - drugs - exert on them. 
The point of view we have adopted here may indeed be considered as complementary to the one of molecular biologists, pharmacologists and systems theoreticians $[77,78]$ who seek to control the cell division cycle at the single cell level by targeted drugs that are hoped-for blockers of intracellular pathways essential in cancer proliferation. Either by targeting a "hub" in the network by a single drug (e.g., imatinib in CML targeting BCR-Abl chimeric tyrosine kinase [126]) or by combining drugs that can hit complementary pathways, they search for "druggable" proteins that can be hit to arrest the cell cycle. A typical and recent example may be found in [122]. However, this approach, obviously valuable to provide new weapons in the war against cancer, by its nature cannot take into account the constraints linked to toxicity or drug resistance issues, which must be considered at the cell population level in a whole-body drug delivery optimisation perspective. This molecular biology approach should also be completed by whole-body pharmacokineticpharmacodynamic molecular modelling to represent the fate of drugs in the organism, as sketched above and in [37, 38]. In other words, these approaches may be thought of as understanding the target and the weapon, whereas, to stay in this metaphor, optimisation of drug control is training to shoot in all conditions to safely reach the target.

Focusing on cell population models, for cancer is never the problem of a single cell, we thus advocate in this chapter the interest of using structured cell population dynamics, to be further integrated in a multiscale setting, in the optimisation of drug delivery in oncology. From intracellular molecular dynamics to human populations, aiming at getting closer to actual clinical applications, we clearly have still hard work ahead, both in modelling and model analysis, and in experimental identification and validation. Various therapeutic optimisation methods have been reviewed in their principles, and we have shown, focusing even more on linear population growth for cancer and for healthy cells, how it is possible to choose one, adapted to the model under consideration. The question of therapeutic optimisation in cancer is vast, and it may be treated in quite different manners, which have to be adapted to the particular clinical problem at stake. Nevertheless, modelling the target, the means of control, and taking account of the known clinical issues, there is still room for mathematical developments to pave the way for optimisation methods that will be able to face always more clinical challenges, all the more so as more links will be developed between mathematicians and clinicians.

\section{Acknowledgments}

Access to data mentioned in Subsection 7.4 has been provided to us by G. van der Horst's lab in Erasmus Medical Centre (Rotterdam, The Netherlands); it was supported by a grant from the European Research Area in Systems Biology (ERASysBio+) and FP7 to the French National Research Agency (ANR) \#ANR-09-SYSB-002-004 for the research network Circadian and Cell 
Cycle Clock Systems in Cancer (C5Sys) coordinated by Francis Lévi (Villejuif, France).

\section{References}

1. Z. Agur, R. Hassin, and S. Levy. Optimizing chemotherapy scheduling using local search heuristics. Oper. Res., 54:829-846, 2006.

2. T. Alarcón, H. Byrne, and P. Maini. A multiple scale model for tumor growth. Multiscale Model Simul, 3:440-475, 2005.

3. A. Altinok, D. Gonze, F. Lévi, and A. Goldbeter. An automaton model for the cell cycle. Interface focus, 1:36-47, 2011.

4. A. Altinok, F. Lévi, and A. Goldbeter. A cell cycle automaton model for probing circadian patterns of anticancer drug delivery. Adv. Drug Deliv. Rev., 59:1036-1010, 2007.

5. A. Altinok, F. Lévi, and A. Goldbeter. Optimizing temporal patterns of anticancer drug delivery by simulations of a cell cycle automaton. In M. Bertau, E. Mosekilde, and H. Westerhoff, editors, Biosimulation in Drug Development, pages 275-297. Wiley-VCH Verlag GmbH \& Co. KGaA, Weinheim, Germany, 2008.

6. A. Altinok, F. Lévi, and A. Goldbeter. Identifying mechanisms of chronotolerance and chronoefficacy for the anticancer drugs 5-fluorouracil and oxaliplatin by computational modeling. Eur. J. Pharm. Sci., 36:20-38, 2009.

7. O. Arino. A survey of structured cell population dynamics. Acta Biotheor., 43:3-25, 1995.

8. O. Arino and M. Kimmel. Comparison of approaches to modeling of cell population dynamics. SIAM J. Appl. Math., 53:1480-1504, 1993.

9. O. Arino and E. Sanchez. A survey of cell population dynamics. J. Theor. Med., 1:35-51, 1997.

10. A. Ballesta, J. Clairambault, S. Dulong, and F. Lévi. Theoretical optimization of irinotecan-based anticancer strategies in case of drug-induced efflux. Appl. Math. Lett., 24:1251-1256, 2011.

11. A. Ballesta, S. Dulong, C. Abbara, B. Cohen, A. Okyar, J. Clairambault, and F. Levi. A combined experimental and mathematical approach for molecular-based optimization of irinotecan circadian delivery. PLoS Comp. Biol., 7:e1002143, 2011.

12. H. Banks, K. L. Sutton, W. C. Thompson, G. Bocharov, M. Doumic, T. Schenkel, J. Argilaguet, S. Giest, C. Peligero, and A. Meyerhans. A new model for the estimation of cell proliferation dynamics using cfse data. $J$. Imunol. Methods, 373:143-160, 2011.

13. D. Barbolosi, A. Benabdallah, F. Hubert, and F. Verga. Mathematical and numerical analysis for a model of growing metastatic tumors. Math Biosci, 218:1-14, 2009.

14. D. Barbolosi and A. Iliadis. Optimizing drug regimens in cancer chemotherapy: a simulation study using a pk-pd model. Comput Biol Med, 31:157-172, 2001.

15. C. Basdevant, J. Clairambault, and F. Lévi. Optimisation of time-scheduled regimen for anti-cancer drug infusion. Mathematical Modelling and Numerical Analysis, 39:1069-1086, 2006. 
16. B. Basse, B. Baguley, E. Marshall, G. Wake, and D. Wall. Modelling the flow cytometric data obtained from unperturbed human tumour cell lines: Parameter fitting and comparison. Bull. Math. Biol., 67:815-830, 2005.

17. B. Basse, B. C. Baguley, E. S. Marshall, W. R. Joseph, B. van Brunt, G. Wake, and D. J. N. Wall. A mathematical model for analysis of the cell cycle in cell lines derived from human tumors. J. Math. Biol., 47:295-312, 2003.

18. B. Basse, B. C. Baguley, E. S. Marshall, W. R. Joseph, B. van Brunt, G. Wake, and D. J. N. Wall. Modelling cell death in human tumour cell lines exposed to the anticancer drug paclitaxel. J Math Biol, 49:329-357, 2004.

19. B. Basse, B. C. Baguley, E. S. Marshall, G. C. Wake, and D. J. N. Wall. Modelling cell population growth with applications to cancer therapy in human tumour cell lines. Prog Biophys Mol Biol, 85:353-368, 2004.

20. B. Basse and P. Ubezio. A generalised age- and phase-structured model of human tumour cell populations both unperturbed and exposed to a range of cancer therapies. Bull. Math. Biol., 69:1673-1690, 2007.

21. F. Bekkal Brikci, J. Clairambault, and B. Perthame. Analysis of a molecular structured population model with polynomial growth for the cell cycle. Mathematical and Computer Modelling, 47:699-713, 2008.

22. F. Bekkal Brikci, J. Clairambault, B. Ribba, and B. Perthame. An age-andcyclin-structured cell population model for healthy and tumoral tissues. Journal of Mathematical Biology, 57:91-110, 2008.

23. S. Bernard, B. Čajavec Bernard, F. Lévi, and H. Herzel. Tumor growth rate determines the timing of optimal chronomodulated treatment schedules. PLoS Computational Biology, 6(3):e1000712, 2010.

24. D. Bertsekas. Nonlinear Programming. Athena Scientific, Nashua, NH, 1995.

25. F. Billy, J. Clairambault, O. Fercoq, S. Gaubert, T. Lepoutre, and T. Ouillon. Proliferation in cell population models with age structure. In Proceedings of ICNAAM 2011, Kallithea Chalkidis (Greece), pages 1212-1215. American Institute of Physics, 2011.

26. F. Billy, J. Clairambault, O. Fercoq, S. Gaubert, T. Lepoutre, T. Ouillon, and S. Saito. Synchronisation and control of proliferation in cycling cell population models with age structure. Math. Comp. Simul., 2012. In press, available on line April 2012.

27. F. Billy, B. Ribba, O. Saut, H. Morre-Trouilhet, T. Colin, D. Bresch, J.-P. Boissel, E. Grenier, and J.-P. Flandrois. A pharmacologically based multiscale mathematical model of angiogenesis and its use in investigating the efficacy of a new cancer treatment strategy. Journal of Theoretical Biology, 260(4):545-562, 2009.

28. S. Bittanti and G. Guardabassi. Optimal periodic control and periodic systems analysis - an overview. In 25th IEEE Conference on Decision and Control, pages 1417-1423, 1986.

29. J. Bonnans, J. Gilbert, C. Lemaréchal, and C. Sagastizábal. Numerical Optimization - Theoretical and Practical Aspects. Universitext. Springer Verlag, Berlin, 2006.

30. D. Bresch, T. Colin, E. Grenier, B. Ribba, and O. Saut. A viscoelastic model for avascular tumor growth. Discrete and Continuous Dynamical Systems, special issue, Sept. 2009, pages 101-108, 2009.

31. D. Bresch, T. Colin, E. Grenier, B. Ribba, and O. Saut. Computational modeling of solid tumor growth: the avascular stage. SIAM Journal on Scientific Computing, 32:2321-2344, 2010. 
32. H. Byrne, T. Alarcón, M. Owen, S. Webb, and P. Maini. Modelling aspects of cancer dynamics: a review. Phil. Trans. Roy. Soc. A, 364:1563-1578, 2006.

33. H. M. Byrne and M. A. Chaplain. Growth of nonnecrotic tumors in the presence and absence of inhibitors. Math Biosci, 130(2):151-181, 1995.

34. H. M. Byrne and M. A. Chaplain. Growth of necrotic tumors in the presence and absence of inhibitors. Math Biosci, 135(15):187-216, 1996.

35. J. Clairambault. Modelling oxaliplatin drug delivery to circadian rhythm in drug metabolism and host tolerance. Adv. Drug Deliv. Rev., 59:1054-1068, 2007.

36. J. Clairambault. A step toward optimization of cancer therapeutics. physiologically based modelling of circadian control on cell proliferation. IEEE-EMB Magazine, 27:20-24, 2008.

37. J. Clairambault. Modelling physiological and pharmacological control on cell proliferation to optimise cancer treatments. Mathematical Modelling of Natural Phenomena, 4:12-67, 2009.

38. J. Clairambault. Optimising cancer pharmacotherapeutics using mathematical modelling and a systems biology approach. Personalized Medicine, 8:271-286, 2011.

39. J. Clairambault, S. Gaubert, and T. Lepoutre. Comparison of Perron and Floquet eigenvalues in age structured cell division models. Mathematical Modelling of Natural Phenomena, 4:183-209, 2009.

40. J. Clairambault, S. Gaubert, and T. Lepoutre. Circadian rhythm and cell population growth. Mathematical and Computer Modelling, 53:1558-1567, 2011.

41. J. Clairambault, M. Hochberg, T. Lorenzi, A. Lorz, and B. Perthame. Populational adaptive evolution and resistance to therapy. Mathematical Modelling and Numerical Analysis, 2012. Accepted.

42. J. Clairambault, B. Laroche, S. Mischler, and B. Perthame. A mathematical model of the cell cycle and its control. Technical report, Number 4892, INRIA, Domaine de Voluceau, BP 105, 78153 Rocquencourt, France, 2003.

43. S. Cui and A. Friedman. Analysis of a mathematical model of the effect of inhibitors on the growth of tumor. Math. Biosci., 164:103-137, 2000.

44. D. Cunningham, Y. Humblet, S. Siena, D. Khayat, H. Bleiberg, A. Santoro, D. Bets, M. Mueser, A. Harstrick, C. Verslype, I. Chau, and E. Van Cutsem. Cetuximab monotherapy and cetuximab plus irinotecan in irinotecanrefractory metastatic colorectal cancer. N. Engl. J. Med., 351:337-345, 2004.

45. A. S. Deakin. Model for the growth of a solid in vitro tumor. Growth, 39(1):159 165,1975

46. A. d'Onofrio. Rapidly acting antitumoral antiangiogenic therapies. Phys Rev E Stat Nonlin Soft Matter Phys, 76(3 Pt 1):031920, 2007.

47. A. d'Onofrio and A. Gandolfi. Tumour eradication by antiangiogenic therapy: analysis and extensions of the model by Hahnfeldt et al. (1999). Math. Biosci., 191:159-184, 2004.

48. A. d'Onofrio and A. Gandolfi. A family of models of angiogenesis and antiangiogenesis anti-cancer therapy. Math Med Biol, 26(1):63-95, 2009.

49. A. d'Onofrio, U. Ledzewicz, H. Maurer, and H. Schättler. On optimal delivery of combination therapy for tumors. Math. Biosci., 222:13-26, 2009.

50. M. Doumic, M. Hoffmann, P. Reynaud, and V.Rivoirard. Nonparametric estimation of the division rate of a size-structured population. SIAM Journal on Numerical Analysis, 50:925-950, March 2012. 
51. M. Doumic, P. Maia, and J. Zubelli. On the calibration of a size-structured population model from experimental data. Acta Biotheoretica, 58:405-413, 2010.

52. M. Doumic, B. Perthame, and J. Zubelli. Numerical solution of an inverse problem in size-structured population dynamics. Inverse Problems, 25:045008 (25pp), 2009.

53. B. Druker, M. Talpaz, D. Resta, B. Peng, E. Buchdunger, J. Ford, N. Lydon, H. Kantarjian, R. Capdeville, S. Ohno-Jones, and C. Sawyers. Efficacy and safety of a specific inhibitor of the bcr-abl tyrosine kinase in chronic myeloid leukemia. N. Engl. J. Med., 344:1031-1037, 2001.

54. P. Dua, V. Dua, and E. Pistikopoulos. Optimal delivery of chemotherapeutic agents in cancer. Comp. Chem. Eng., 32:99-107, 2008.

55. A. Ergun, K. Camphausen, and L. M. Wein. Optimal scheduling of radiotherapy and angiogenic inhibitors. Bull Math Biol, 65(3):407-424, 2003.

56. K. R. Fister and J. C. Panetta. Optimal control applied to cell-cycle-specific cancer chemotherapy. SIAM Journal on Applied Mathematics, 60(3):1059 1072, 2000.

57. H. B. Frieboes, M. E. Edgerton, J. P. Fruehauf, F. R. A. J. Rose, L. K. Worrall, R. A. Gatenby, M. Ferrari, and V. Cristini. Prediction of drug response in breast cancer using integrative experimental/computational modeling. Cancer Res, 69:4484-4492, 2009.

58. R. Gatenby. A change of strategy in the war on cancer. Nature, 459:508-509, 2009.

59. R. Gatenby and E. Gawlinski. A reaction-diffusion model of cancer invasion. Cancer Research, 56:5745-5753, 1996.

60. R. Gatenby, P. K. Maini, and E. Gawlinski. Analysis of tumor as an inverse problem provides a novel theoretical framework for understanding tumor biology and therapy. Applied Mathematics Letters, 15:339-345, 2002.

61. R. Gatenby, A. Silva, R. Gillies, and B. Friden. Adaptive therapy. Cancer Research, 69:4894-4903, 2009.

62. B. Gompertz. On the nature of the function expressive of the law of human mortality, and on a new mode of determining the value of life contingencies. Philos. Transact. of the Royal Society of London, 115:513-585, 1825.

63. H. Greenspan. Models for the growth of a solid tumor by diffusion. Stud. Appl. Math., 52:317-340, 1972.

64. M. Gyllenberg, A. Osipov, and L. Päivärinta. The inverse problem of linear age-structured population dynamics. J. Evol. Equations, 2:223-239, 2002.

65. M. Gyllenberg and G. F. Webb. A nonlinear structured population model of tumor growth witsh quiescence. J Math Biol, 28:671-694, 1990.

66. T. Haferlach. Molecular genetic pathways as therapeutic targets in acute myeloid leukemia. Hematology, 2008:400-411, 2008. Am. Soc. Hematol. Educ. Program.

67. P. Hahnfeldt, D. Panigrahy, J. Folkman, and L. Hlatky. Tumor development under angiogenic signaling: a dynamical theory of tumor growth, treatment response, and postvascular dormancy. Cancer Res, 59:4770-4775, 1999.

68. N. Hansen. The CMA evolution strategy: A comparing review. In J. Lozano, P. Larraaga, I. Inza, and E. Bengoetxea, editors, Towards a new evolutionary computation. Advances in estimation of distribution algorithms, pages 75-102. Springer, 2006. 
69. P. Hinow, S. Wang, C. Arteaga, and G. Webb. A mathematical model separates quantitatively the cytostatic and cytotoxic effects of a her2 tyrosine kinase inhibitor. Theoretical Biology and Medical Modelling, 4:14, 2007. doi:10.1186/1742-4682-4-14.

70. A. Iliadis and D. Barbolosi. Optimizing drug regimens in cancer chemotherapy by an efficacy-toxicity mathematical model. Comput Biomed Res, 33:211-226, 2000 .

71. K. Iwata, K. Kawasaki, and N. Shigesada. A dynamical model for the growth and size distribution of multiple metastatic tumors. J Theor Biol, 203:177-186, 2000 .

72. T. Jackson. Intracellular accumulation and mechanism of action of doxorubicin in a spatio-temporal tumor model. J. Theor. Biol., 220:201-213, 2003.

73. T. Jackson and H. Byrne. A mathematical model to study the effects of drug resistance and vasculature on the response of solid tumors to chemotherapy. Mathematical Biosciences, 164:17-38, 2000.

74. Y. Kheifetz, Y. Kogan, and Z. Agur. Long-range predictability in models of cell populations subjected to phase-specific drugs: growth-rate approximation using properties of positive compact operators. Math. Models Methods Appl. Sci., 16:1155-1172, 2006.

75. M. Kimmel and A. Świerniak. Control theory approach to cancer chemotherapy: Benefiting from phase dependence and overcoming drug resistance. In A. Friedman, editor, Tutorials in Mathematical Biosciences III, volume 1872 of Lecture Notes in Mathematics, pages 185-221. Springer Berlin / Heidelberg, 2006.

76. A. Kirsch. An Introduction to the Mathematical Theory of Inverse Problems. Springer, New York, 2nd edition, 2011.

77. H. Kitano. Cancer as a robust system: Implications for anticancer therapy. Nature Rev. Cancer, 3:227-235, 2004.

78. H. Kitano. A robustness-based approach to systems-oriented drug design. $\mathrm{Na}$ ture Rev. Drug Discovery, 6:202-210, 2007.

79. M. Kohandel, M. Kardar, M. Milosevic, and S. Sivaloganathan. Dynamics of tumor growth and combination of anti-angiogenic and cytotoxic therapies. Phys Med Biol, 52:3665-3677, 2007.

80. F. Kozusko, P. Chen, S. G. Grant, B. W. Day, and J. C. Panetta. A mathematical model of in vitro cancer cell growth and treatment with the antimitotic agent curacin a. Math Biosci, 170(1):1-16, 2001.

81. A. Laird. Dynamics of tumour growth. Br. J. Cancer, 13:490-502, 1964.

82. A. Lasota and M. Mackey. Chaos, Fractals, and Noise: Stochastic Aspects of Dynamics. Springer, New York, 2nd edition, 1994.

83. U. Ledzewicz, H. Maurer, and H. Schaettler. Optimal and suboptimal protocols for a mathematical model for tumor anti-angiogenesis in combination with chemotherapy. Mathematical Biosciences and Engineering, 8:307-323, 2011.

84. U. Ledzewicz, H. Maurer, and H. Schaettler. Optimal and suboptimal protocols for a mathematical model for tumor anti-angiogenesis in combination with chemotherapy. Mathematical Biosciences and Engineering, 8(2):307-323, 2011.

85. U. Ledzewicz and H. Schttler. Optimal controls for a model with pharmacokinetics maximizing bone marrow in cancer chemotherapy. Math Biosci, 206:320-342, 2007.

86. E. Lehmann and G. Casella. Theory of point estimation. Springer Texts in Statistics, 2nd edition, 1998. 
87. F. Lévi. Cancer chronotherapeutics. Special issue of Chronobiology International, 19:1-19, 2002.

88. F. Lévi. Chronotherapeutics: the relevance of timing in cancer therapy. Cancer Causes Control, 17:611-621, 2006.

89. F. Lévi. The circadian timing system: A coordinator of life processes. implications for the rhythmic delivery of cancer therapeutics. IEEE-EMB Magazine, 27:17-20, 2008.

90. F. Lévi, A. Altinok, J. Clairambault, and A. Goldbeter. Implications of circadian clocks for the rhythmic delivery of cancer therapeutics. Phil. Trans. Roy. Soc. A, 366:3575-3598, 2008.

91. F. Lévi, A. Okyar, S. Dulong, P. Innominato, and J. Clairambault. Circadian timing in cancer treatments. Annual Review of Pharmacology and Toxicology, 50:377-421, 2010.

92. F. Lévi and U. Schibler. Circadian rhythms: Mechanisms and therapeutic implications. Ann. Rev. Pharmacol. Toxicol., 47:493-528, 2007.

93. L. Ljung. System Identification - Theory for the User. PTR Prentice Hall, Upper Saddle River, N.J., 2nd edition, 1999.

94. M. Lupi, P. Cappella, G. Matera, C. Natoli, and P. Ubezio. Interpreting cell cycle effects of drugs: the case of melphalan. Cancer Chemother Pharmacol, 57:443-457, 2006.

95. M. Lupi, G. Matera, D. Branduardi, M. D'Incalci, and P. Ubezio. Cytostatic and cytotoxic effects of topotecan decoded by a novel mathematical simulation approach. Cancer Res, 64:2825-2832, 2004.

96. R. Martin. Optimal control drug scheduling of cancer chemotherapy. Automatica, 28:1113-1123, 1992.

97. R. B. Martin, M. E. Fisher, R. F. Minchin, and K. L. Teo. Low-intensity combination chemotherapy maximizes host survival time for tumors containing drug-resistant cells. Math Biosci, 110:221-252, 1992.

98. R. B. Martin, M. E. Fisher, R. F. Minchin, and K. L. Teo. Optimal control of tumor size used to maximize survival time when cells are resistant to chemotherapy. Math Biosci, 110:201-219, 1992.

99. H. Maurer, C. Büskens, J.-H. R. Kim, and C. Y. Kaya. Optimization methods for the verification of second order sufficient conditions for bang-bang controls. Optimal Control Applications and Methods, 26(3):129-156, 2005.

100. M. J. Mauro, M. O'Dwyer, M. C. Heinrich, and B. J. Druker. STI571: A paradigm of new agents for cancer therapeutics. J. Clin. Oncol., 20:325-334, 2002.

101. D. McElwain and P. Ponzo. A model for the growth of a solid tumor with non-uniform oxygen consumption. Math. Biosci., 35:267-279, 1977.

102. A. McKendrick. Applications of mathematics to medical problems. Proc. Edinburgh Math. Soc., 54:98-130, 1926.

103. J. Metz and O. Diekmann. The dynamics of physiologically structured populations, volume 68 of Lecture notes in biomathematics. Springer, New York, 1986.

104. F. Montalenti, G. Sena, P. Cappella, and P. Ubezio. Simulating cancer-cell kinetics after drug treatment: application to cisplatin on ovarian carcinoma. Phys Rev E, 57:5877-5887, 1998.

105. D. Morgan. The Cell Cycle: Principles of Control. Primers in Biology series. Oxford University Press, 2006. 
106. J. Murray. Optimal control for a cancer chemotherapy problem with general growth and loss functions. Math Biosci, 98:273-287, 1990.

107. J. Murray. Some optimal control problems in cancer chemotherapy with a toxicity limit. Math Biosci, 100:49-67, 1990.

108. J. Murray. The optimal scheduling of two drugs with simple resistance for a problem in cancer chemotherapy. IMA J Math Appl Med Biol, 14:283-303, 1997.

109. J. Murray. Mathematical biology. I: An introduction. Springer, 3rd edition, 2002.

110. J. Murray. Mathematical biology. II: Spatial models and biomedical applications. Springer, 3rd edition, 2003.

111. J. Nocedal and S. Wright. Numerical Optimization. Springer, New York, 1999.

112. E. Norris, J. King, and H. Byrne. Modelling the response of spatially structured tumours to chemotherapy: Drug kinetics. Mathematical and Computer Modelling, 43:820-837, 2006.

113. S. Nowsheen, K. Aziz, M. I. Panayiotidis, and A. G. Georgakilas. Molecular markers for cancer prognosis and treatment: have we struck gold? Cancer Letters, 2011. Published on line, November 2011, DOI:10.1016/j.canlet.2011.11.022.

114. J. Panetta and J. Adam. A mathematical model of cell-specific chemotherapy. Math Comput Modelling, 22:67, 1995.

115. J. C. Panetta. A mathematical model of breast and ovarian cancer treated with paclitaxel. Math. Biosci., 146(2):89-113, 1997.

116. J. C. Panetta, W. E. Evans, and M. H. Cheok. Mechanistic mathematical modelling of mercaptopurine effects on cell cycle of human acute lymphoblastic leukaemia cells. Br J Cancer, 94(1):93-100, 2006.

117. J. C. Panetta, M. N. Kirstein, A. J. Gajjar, G. Nair, M. Fouladi, and C. F. Stewart. A mechanistic mathematical model of temozolomide myelosuppression in children with high-grade gliomas. Math Biosci, 186:29-41, 2003.

118. F. Pereira, C. Pedreira, and J. de Sousa. A new optimization based approach to experimental combination chemotherapy. Frontiers Med. Biol. Engng., 6(4):257-268, 1995.

119. B. Perthame. Transport Equations in Biology. Frontiers in Mathematics series. Birkhäuser, Boston, 2007.

120. B. Perthame and J. Zubelli. On the inverse problem for a size-structured population model. Inverse Problems, 23:1037-1052, 2007.

121. L. S. Pontryagin, V. G. Boltyanski, R. V. Gamkrelidze, and E. F. Mishchenko. The mathematical theory of optimal processes. Interscience Publishers, 1962. Translated from the Russian by K.N. Trirogoff.

122. H. Prenen, S. Tejpar, and E. V. Cutsem. New strategies for treatment of kras mutant metastatic colorectal cancer. Clin. Cancer Res., 16:2921-2926, 2010.

123. B. Ribba, O. Saut, T. Colin, D. Bresch, E. Grenier, and J. P. Boissel. A multiscale mathematical model of avascular tumor growth to investigate the therapeutic benefit of anti-invasive agents. J Theor Biol, 243:532-541, 2006.

124. B. Ribba, E. Watkin, M. Tod, P. Girard, E. Grenier, B. You, E. Giraudo, and G. Freyer. A model of vascular tumour growth in mice combining longitudinal tumour size data with histological biomarkers. Eur J Cancer, 47:479-490, 2011.

125. A. Sakaue-Sawano, K. Ohtawa, H. Hama, M. Kawano, M. Ogawa, and A. Miyawaki. Tracing the silhouette of individual cells in S/G2/M phases with fluorescence. Chem Biol., 15:1243-48, 2008. 
126. N. Shah, C. Tran, F. Lee, P. Chen, D. Norris, and C. Sawyers. Overriding imatinib resistance with a novel ABL kinase inhibitor. Science, 305:399-401, 2004.

127. R. M. Shymko. Cellular and geometric control of tissue growth and mitotic instability. J Theor Biol, 63(2):355-374, 1976.

128. J. P. Sinek, S. Sanga, X. Zheng, H. B. Frieboes, M. Ferrari, and V. Cristini. Predicting drug pharmacokinetics and effect in vascularized tumors using computer simulation. J Math Biol, 58:485-510, 2009.

129. J. C. Spall. Introduction to stochastic search and optimization: estimation, simulation, and control. John Wiley \& Sons, Ltd., 2003.

130. L. Spinelli, A. Torricelli, P. Ubezio, and B. Basse. Modelling the balance between quiescence and cell death in normal and tumour cell populations. Math Biosci, 202:349-370, 2006.

131. K. Swanson, E. Alvord, and J. Murray. A quantitative model for differential motility of gliomas in grey and white matter. Cell Prolif., 33:317-329, 2000.

132. K. Swanson, E. Alvord, and J. Murray. Quantifying efficacy of chemotherapy of brain tumors with homogeneous and heterogeneous drug delivery. $J$. Neurosurg., 50:223-237, 2002.

133. K. R. Swanson, E. C. Alvord, and J. D. Murray. Virtual brain tumours (gliomas) enhance the reality of medical imaging and highlight inadequacies of current therapy. Br J Cancer, 86:14-18, 2002.

134. A. Swierniak, A. Polanski, and M. Kimmel. Optimal control problems arising in cell-cycle-specific cancer chemotherapy. Cell Prolif, 29:117-139, 1996.

135. P. Ubezio, M. Lupi, D. Branduardi, P. Cappella, E. Cavallini, V. Colombo, G. Matera, C. Natoli, D. Tomasoni, and M. D'Incalci. Quantitative assessment of the complex dynamics of G1, S, and G2-M checkpoint activities. Cancer Res, 69:5234-5240, 2009.

136. M. Villasana, G. Ochoa, and S. Aguilar. Modeling and optimization of combined cytostatic and cytotoxic cancer chemotherapy. Artificial Intelligence in Medicine, 50(3):163-173, 2010.

137. E. Walter and L. Pronzato. Identification of Parametric Models from Experimental Data. Springer, Communications and control engineering series, London, 2nd edition, 1997.

138. G. Webb. Resonance phenomena in cell population chemotherapy models. Rocky Mountain J. Math, 20(4):1195-1216, 1990.

139. G. Webb. A cell population model of periodic chemotherapy treatment. Biomedical Modeling and Simulation, pages 83-92, 1992.

140. G. Webb. A non linear cell population model of periodic chemotherapy treatment. Recent Trends Ordinary Differential Equations, Series in Applicable Analysis 1, pages 569-583, 1992.

141. X. Zheng, S. M. Wise, and V. Cristini. Nonlinear simulation of tumor necrosis, neo-vascularization and tissue invasion via an adaptive finite-element/level-set method. Bull Math Biol, 67:211-259, 2005. 